\title{
Engineered E. coli for the targeted deposition of therapeutic payloads to sites of disease
}

Jason Lynch

Massachusetts General Hospital

Coral Coral González-Prieto

Massachusetts General Hospital

Analise Reeves

Synlogic

Urmila Powale

Tufts University

Neha Godbole

Massachusetts General Hospital

Jacqueline Tremblay

Tufts Cummings School of Veterinary

Florian Schmidt

University of Bonn https://orcid.org/0000-0002-9979-9769

Hidde Ploegh

Boston Children's Hospital https://orcid.org/0000-0002-1090-6071

Jonathan Glickman

Beth Israel Deaconess Medical Center

John Leong

Tufts University

Charles Shoemaker

Tufts Cummings School of Veterinary

Wendy Garrett

Harvard University https://orcid.org/0000-0002-5092-0150

CAMMIE LESSER ( $\nabla$ clesser@mgh.harvard.edu )

Massachusetts General Hospital

\section{Article}

Keywords:

Posted Date: January 21st, 2022 
DOI: https://doi.org/10.21203/rs.3.rs-1233122/v1

License: (a) (i) This work is licensed under a Creative Commons Attribution 4.0 International License. Read Full License 
2 \#Jason P. Lynch"1,2, "Coral González-Prieto ${ }^{1,2}$, Analise Z. Reeves ${ }^{1,2}$, Urmila Powale ${ }^{1,2}$, Neha

3 Godbole $^{1,2}$, Jacqueline M. Tremblay ${ }^{3}$, Florian I. Schmidt ${ }^{4}$, Hidde L. Ploegh ${ }^{5}$, Jonathan N.

4 Glickman $^{6,7}$, John M. Leong ${ }^{8}$, Charles B. Shoemaker ${ }^{3}$, Wendy S. Garrett ${ }^{9,10,11}$, *Cammie F.

$5 \quad$ Lesser $^{1,2,11}$

$6{ }^{1}$ Center for Bacterial Pathogenesis, Division of Infectious Diseases, Department of Medicine,

7 Massachusetts General Hospital, MA 02115, USA.

$8{ }^{2}$ Department of Microbiology, Blavatnik Institute, Harvard Medical School, Boston, MA 02115, 9 USA.

$10{ }^{3}$ Department of Infectious Disease and Global Health, Tufts Cummings School of Veterinary

11 Medicine, North Grafton, Massachusetts, MA 01536, USA

$12{ }^{4}$ Institute of Innate Immunity, Medical Faculty, University of Bonn, 53127 Bonn, Germany.

$13{ }^{5}$ Boston Children's Hospital and Harvard Medical School, Boston, MA 02115, USA

$14{ }^{6}$ Department of Pathology, Harvard Medical School, Boston, MA 02115, USA.

$15 \quad{ }^{7}$ Beth Israel Deaconess Medical Center, Boston, MA 02215-5400, USA.

$16{ }^{8}$ Department of Molecular Biology and Microbiology, Tufts University School of Medicine,

17 Boston, MA, 02111, USA.

$18{ }^{9}$ Departments of Immunology and Infectious Diseases and Harvard T. H. Chan Center for the

19 Microbiome in Public Health, Harvard T.H. Chan School of Public Health, Boston, MA 02115, 20 USA. 
$21{ }^{10}$ Department of Medical Oncology, Dana-Farber Cancer Institute, Boston, MA 02215, USA.

$22{ }^{11}$ Broad Institute of MIT and Harvard, Cambridge, MA 02142, USA.

23 "These authors contributed equally

$24 \quad{ }^{*}$ Correspondence: clesser@mgh.harvard.edu (C.F. Lesser)

25

26

27

28 
30 New drug platforms are needed which enable the directed delivery of therapeutics to sites of

31 disease to maximize efficacy and limit off-target effects. Here, we report the development of

$32 \mathrm{PROT}_{3} \mathrm{EcT}$, commensal Escherichia coli engineered for the direct secretion of proteins into their

33 surroundings. $\mathrm{PROT}_{3} \mathrm{EcT}$ are composed of four modular components: an E. coli chassis, a

34 modified bacterial protein secretion system, a regulatable transcriptional activator, and a

35 secretable therapeutic payload. $\mathrm{PROT}_{3} \mathrm{EcT}$ that secrete functional single-domain antibodies,

36 nanobodies $(\mathrm{Nb})$, stably colonize and maintain a functional secretion system within the

37 intestines of mice. A single prophylactic dose of $\mathrm{PROT}_{3} \mathrm{EcT}$ that secretes a tumor necrosis

38 factor alpha (TNFa) neutralizing $\mathrm{Nb}$ is sufficient to ablate TNF levels and prevent the

39 development of injury and inflammation in a chemically-induced model of inflammatory bowel

40 disease. This work lays the foundation for the development of $\mathrm{PROT}_{3} \mathrm{EcT}$ as a therapeutic

41 platform for the treatment of at least gastrointestinal-based diseases.

\section{Introduction}

44 Microbe-based therapeutics are emerging as a platform for the development of interventions for

45 the treatment of a variety of diseases, particularly those with etiologies linked to the gut. In

46 addition to searching for cocktails of beneficial natural isolates, synthetic biology-based

47 approaches are being used to engineer microbes with additional therapeutic capabilities,

48 including the targeted deposition of therapeutic payloads to sites of disease. Due to their ease

49 of production, administration, and natural capacity to synthesize and deliver complex biologics,

50 engineered microorganisms hold enormous potential as affordable options to traditional biologic

51 therapies. In addition, by outfitting them with high specificity payloads, they provide a platform

52 for the development of interventions with improved efficacy and limited off-target effects. 
Escherichia coli Nissle 1917 (EcN), a probiotic with GRAS (generally recognized as

54 safe) status ${ }^{1}$, is gaining traction as a chassis for synthetic biology. EcN has inherent anti-

55 bacterial and anti-inflammatory activities and is genetically tractable. A variety of strategies are

56 being pursued to enhance its therapeutic potential. For example, variants with enhanced

57 metabolic capabilities are being investigated for removal of toxic intermediates associated with

58 metabolic diseases ${ }^{2-4}$. Similarly, efforts are underway to develop variants that deliver therapeutic

59 payloads to sites of disease. However, given the difficulty with engineering Gram-negative

60 bacteria to secrete proteins into their surroundings, work has primarily focused on developing

61 variants of EcN programmed to release intracellular cargo ${ }^{5-7}$ as well as to express outer

62 membrane adhesins ${ }^{8}$ and Curli modified to display proteins of interest on the bacterial surface ${ }^{9}$.

The general secretion $(\mathrm{Sec})$ and twin arginine translocation (Tat) pathways, common to

64 both Gram-negative and Gram-positive bacteria, are the main systems by which both transport

65 proteins across their cytosolic membranes ${ }^{9}$. These systems promote the deposition of proteins

66 into the surroundings of Gram-positive bacteria, but only into the outer membrane of their Gram-

67 negative relatives. Only a small subset of periplasmic proteins are targeted for secretion across

68 their outer membrane into the surroundings (for review, see ${ }^{10}$ ). However, numerous Gram-

69 negative bacterial pathogens utilize complex nanomachines, including type III secretions

70 systems (T3SSs), to transport bacterial proteins directly into the cytosol of host cells. The fully

71 assembled type III secretion apparatus (T3SA) is embedded within the outer envelope of the

72 bacterium with a needle-like extension that docks onto and forms pores in host cell membranes.

73 We previously established that the T3SA of Shigella flexneri is functional when introduced into

74 laboratory strains of $E$. coli $i^{1-14}$.

75 Here, we report the development of $\mathrm{PROT}_{3} \mathrm{EcT}$ (PRObiotic $\underline{\text { Iype } \underline{3}}$ secretion $\underline{\text { E. }} \underline{\text { coli }}$

76 Therapeutic), E. coli engineered with a Shigella T3SA modified to secrete proteins into its

77 surroundings, as opposed to directly into eukaryotic cells. When fused to an $\mathrm{N}$-terminal type III 
secretion sequence, fully functional camelid single-domain antibodies (also known as nanobodies or $\mathrm{VHH}$ ), are secreted by $\mathrm{PROT}_{3} \mathrm{EcT}$. PROT $\mathrm{PRcT}_{3}$ is modular in design, composed of four elements: (1) an E. coli strain, (2) the modified T3SA, (3) its master transcriptional

81 regulator (VirB), and (4) a therapeutic payload (Fig. 1a). PROT $_{3} E_{c T}$-4, a variant of PROT $_{3} \mathrm{EcT}$

82 engineered such that all components are maintained in the absence of antibiotic selection, is

83 unimpaired in growth and capable of colonizing the intestines of mice for at least 14 days. In

84 support of the therapeutic potential of the $\mathrm{PROT}_{3} \mathrm{EcT}$ platform, TNF-PROT 3 EcT, a variant of

$85 \mathrm{PROT}_{3}$ EcT-4 engineered to secrete an anti-TNFa nanobody, is as effective as systemically

86 administered anti-TNFa monoclonal antibodies in suppressing the development of inflammation

87 in a chemically induced preclinical model of inflammatory bowel disease. Together, these

88 studies provide the foundation for the further development of $\mathrm{PROT}_{3} \mathrm{EcT}$ as a versatile

89 therapeutic platform (Fig. 1b).

\section{$90 \quad$ Results}

\section{Development of $\mathrm{PROT}_{3} \mathrm{EcT}$}

92 The genes that encode the 20 components that form the Shigella T3SA are contained within

93 the adjacent Ipa, Mxi, and Spa operons on a large virulence plasmid ${ }^{15,16}$. The Mxi and Spa

94 operons encode all of the structural components needed to form the T3SA. The Ipa operon

95 encodes the proteins that form the tip complex that holds the machine in an OFF configuration

96 prior to host cell contact and a pore complex in the host cell membrane upon which the machine

97 docks before injecting proteins into host cells ${ }^{17-19}$. We previously described a recombineering-

98 based platform to transfer large regions of this virulence plasmid into defined engineered

99 synthetic loci on the E. coli chromosome ${ }^{11-13,20}$. Using this technology, we developed laboratory

100 strains of E. coli that encode and express the Ipa, Mxi, and Spa operons capable of delivering

101 heterologous proteins into mammalian cells ${ }^{14}$. 
With the goal of developing E. coli that efficiently secrete proteins into their surroundings

103 (Fig. S1), we compared the secretory activity of DH10b E. coli that contain the Ipa, Mxi and Spa 104 operons versus the Mxi and Spa operons, each inserted at a single defined chromosomal locus. 105 Each strain was transformed with a low-copy number plasmid that expresses VirB, the shared 106 transcription factor of the Mxi, Spa and Ipa operons (Fig. S1a), under the control of the IPTG 107 (isopropyl $\beta$ - d-1-thiogalactopyranoside)-inducible Ptrc promoter. The resulting strains are 108 referred to here as mT3Ec_Ipa-Mxi-Spa and mT3Ec_Mxi-Spa (Fig. S1b).

When grown under conditions that promote T3SA expression and exposed to Congo 110 red, a dye that triggers secretion in the absence of host cells ${ }^{21}$, mT3Ec_Ipa-Mxi-Spa and 111 mT3Ec_Mxi-Spa, secreted similar levels of IPTG-inducible OspC2 (a native Shigella T3SA 112 secreted protein), demonstrating that the absence of the Ipa operon has no effect on the activity 113 of the T3SA (Fig. 2a). To ensure that OspC2 detected in the supernatant fractions was not due 114 to bacterial cell lysis, we also monitored for the presence of GroEL, a highly abundant cytosolic 115 protein. As expected, GroEL was detected in the intact bacteria, but not in the supernatant 116 factions (Fig. 2a). Furthermore, in the absence of Congo red, OspC2 was abundantly secreted 117 by mT3Ec_Mxi-Spa, but not mT3Ec_Ipa-Mxi-Spa, demonstrating that mT3Ec_Mxi-Spa 118 constitutively secretes proteins into its surroundings (Fig. 2b). When we examined the full set of 119 proteins present in the supernatant of mT3Ec_Mxi-Spa, OspC2 was the most abundantly 120 secreted bacterial protein, establishing that the introduction of the Mxi-Spa operons and VirB is 121 sufficient to outfit DH10b E. coli with a robust IPTG-inducible secretion system (Fig. 2c).

We next investigated whether similar modifications to two non-pathogenic human $E$. coli 123 isolates would similarly equip these strains with a functional protein secretion system. First, we 124 developed $\mathrm{PROT}_{3}$ EcT-1, E. coli Nissle $1917(\mathrm{EcN})$ engineered with the Mxi-Spa operons at the 125 analogous chromosomal locus as mT3Ec_Mxi-Spa. To test whether PROT 3 EcT-1 assembles a 126 functional T3SA, we introduced plasmids encoding IPTG-inducible virB and ospC2 (Fig. 2d) and 
127 monitored OspC2 secretion following induction of expression of both. VirB-expressing

$128 \mathrm{PROT}_{3} \mathrm{EcT}-1$, like mT3Ec_Mxi-Spa, secretes OspC2 (Fig. 2e), albeit at somewhat lower levels.

129 Similar modifications to the E. coli human isolate $\mathrm{HS}$, led to the generation of $\mathrm{PROT}_{3} \mathrm{EcT}-2$,

130 which secreted OspC2 at levels closer to that of mT3Ec_Mxi-Spa (Fig. S1c), suggesting that the

131 Mxi-Spa T3SA platform will function similarly when introduced into additional E. coli strains.

To mimic the in vivo situation more closely, and to assess whether the strains have extended secretory activity, we followed the levels of OspC2 in the supernatant fractions of

134 mT3Ec_Mxi-Spa and $\mathrm{PROT}_{3} \mathrm{EcT}-1$ when grown in media that supports their growth. Increasing

135 levels of secreted OspC2, but not GroEL, was observed over a 6-hour time course (Fig. 2f).

Lastly, Shigella are intracellular pathogens that rely on their T3SS and its secreted

137 proteins to invade non-phagocytic epithelial cells. Given that bacteria engineered with the Mxi-

138 Spa operons lack effectors, they are not expected to invade host cells. We therefore compared

139 the ability of Shigella, $\mathrm{PROT}_{3} \mathrm{EcT}-1, \mathrm{mT} 3 \mathrm{Ec} \_\mathrm{Mx}-\mathrm{Spa}$, DH10b E. coli and EcN to invade cells

140 using a gentamicin protection assay. As expected, Shigella, but none of the other strain were

141 observed within epithelial cells (Fig. 2g).

$142 \mathrm{PROT}_{3} \mathrm{EcT}$ can be engineered to secrete nanobodies

143 Nanobodies $(\mathrm{Nb})$, the $\sim 15 \mathrm{kDa}$ variable domains of heavy chain-only antibodies, are ideal

144 substrates from our bacterial secretion system as they are small stable proteins that generally

145 exhibit strong antigen-binding affinity. We previously found that fusion of the first $\sim 50 \mathrm{~N}$-terminal

146 amino acids of numerous Shigella type III effectors to heterologous proteins is sufficient to

147 generate variants secreted by mT3Ec_Ipa-Mxi-Spa ${ }^{12,14}$. Thus, we screened for modifications to

148 a representative $\mathrm{Nb}$ that result in its recognition by $\mathrm{PROT}_{3} \mathrm{EcT}$. Nb ${ }^{\mathrm{ASC}}{ }^{22}$ was fused to the first 50

149 residues of eight Shigella effectors (IpaH4.5, IpaH7.8, IpaH9.8, OspE, OspF, OspD3, VirA, and

150 OspG). We hereafter refer to these regions as secretion sequences. $\mathrm{Nb}^{\mathrm{ASC}}$ fused to the OspC2 
151 or OspG secretion sequences resulted in the highest level of secretion (Fig. 3a). Fusion of the

152 OspC2 secretion sequence also resulted in secretion of monomeric $\mathrm{Nb}^{\mathrm{PD} L 1}{ }^{23}, \mathrm{Nb}^{\mathrm{CTLA4}}{ }^{24}, \mathrm{Nb}^{\mathrm{NPI}}$ $153{ }^{25}$, and $\mathrm{Nb}^{\mathrm{St} \times 2} 26$ as well as heterodimeric and heterotrimeric $\mathrm{Nb}^{\mathrm{St} \times 2}$ (Fig. 3b, c). As observed with

154 OspC2 (Fig. 2c), Nbs were the most abundant protein present in the supernatants of $155 \mathrm{PROT}_{3} \mathrm{EcT}-1$ (Fig. 3d).

In parallel, we investigated whether some of the native E. coli secretion systems that are 157 currently explored for secretion of recombinant proteins $\mathrm{s}^{27-30}$ can also be adapted to secrete Nbs. 158 In these systems, the proteins are secreted via a 2-step process. Post-delivery into the 159 periplasm via the Sec system, they are secreted across the outer membrane via unknown 160 pathway(s). We fused $\mathrm{Nb}^{\mathrm{St} \times 2}$ to full length E. coli OsmY, E. coli YebF and Bacillus Cel-CD, as 161 well as the first 20 residues of Cel-CD, which encodes its secretion signal sequence. Only 162 fusion to YebF resulted in a secreted $\mathrm{Nb}$, which was present at much lower levels in the 163 supernatants of EcN as compared to the same $\mathrm{Nb}$ in the supernatants of $\mathrm{PROT}_{3} \mathrm{EcT}$ (Fig. S2a).

164 We were unable to detect expression of the Cel-CD fusions, which reflected a lack of 165 expression rather than a deficiency of our detection method because the expression and 166 secretion of Cel-CD fusion proteins was detectable when expressed in protease-deficient BL21 167 E. coli (Fig. S2b), consistent with published studies ${ }^{29,30}$. Thus, at least for the candidate $\mathrm{Nb}$ 168 studied, the $\mathrm{PROT}_{3} \mathrm{EcT}$ platform vastly outperformed the native $E$. coli secretion systems. 169

\section{Development of constitutively active $\mathrm{Nb}$-secreting PROT ${ }_{3} \mathrm{EcT}$}

Before studying $\mathrm{PROT}_{3} \mathrm{EcT}$ in mouse models of disease, we sought to generate a

172 variant that constitutively secretes Nbs and maintains all its genetically engineered components

173 in the absence of antibiotic selection. For VirB, we first replaced its IPTG-inducible Ptrc

174 promoter with ones predicted to be constitutively active in the gut, Shigella PvirF ${ }^{31}$, E. coli

$175 \mathrm{PompC}^{32}$, and two synthetic promoters, BBa_J23115 and BBa_J23119 ${ }^{33}$. PROT ${ }_{3}$ EcT-1 variants 
carrying plasmids that encode each of these constitutively active VirB secreted Nbs at levels as

177 the variant expressing IPTG-inducible VirB (Fig. 4a). Thus, we introduced the PJ23119-virB

178 expression cassette into the chromosome of $\mathrm{PROT}_{3} \mathrm{EcT}-1$. The resulting strain, $\mathrm{PROT}_{3} \mathrm{EcT}-3$

179 (Fig. 4b), secreted $\mathrm{Nb}$ at levels equivalent to that of the parent strain that encodes PJ23119-virB 180 on a plasmid (Fig. 4c).

For the $\mathrm{Nb}$ expression cassette, we generated a variant that was constitutively expressed by replacing its Ptrc promoter with the constitutive BBa_PJ23108 promoter (Fig. 4d).

183 To maintain flexibility in terms of introducing payload expression circuits and to enable higher

184 levels of expression, rather than introduce these circuits onto the chromosome of $\mathrm{PROT}_{3} \mathrm{EcT}$, 185 we chose to encode them on a plasmid. To ensure plasmid maintenance in the absence of 186 antibiotic pressure, we built on prior work from Hwang and colleagues ${ }^{34}$ and developed $187 \mathrm{PROT}_{3} \mathrm{EcT}-4$, a derivative that lacks alr and dadX (Fig 4e). These genes encode EcN's two 188 alanine racemases that convert $L$ - to $D$-alanine, an amino acid that is essential for cell wall 189 biosynthesis that is very limited in the mammalian GI tract ${ }^{35}$. We then inserted an intact alr gene 190 onto the Nb-producing plasmid to facilitate in vivo pressure for plasmid maintenance.

$191 \mathrm{PROT}_{3} \mathrm{EcT}-3$ and $\mathrm{PROT}_{3} \mathrm{EcT}-4$ that carry this plasmid secreted similar levels of $\mathrm{Nbs}$, but 192 whereas Nb-production by $\mathrm{PROT}_{3} \mathrm{EcT}-3$ is lost in the absence of antibiotic selection, $193 \mathrm{PROT}_{3}$ EcT-4 stably maintains production (Fig. 4f, Fig. S3a). PROT 3 EcT-4 and unmodified EcN 194 exhibited essentially identical growth patterns, regardless of whether the bacteria expressed 195 and secreted Nbs (Fig. 4g), indicating that these modifications do not add a significant metabolic 196 burden.

197 PROT ${ }_{3}$ EcT stably colonizes the gastrointestinal tract of mice.

Our initial in vivo experiments focused on investigating the ability of $\mathrm{Nb}$-secreting $199 \mathrm{PROT}_{3} \mathrm{EcT}-4$ to colonize the mouse gastrointestinal tract while maintaining a functional 
secretion system. We first monitored the levels of bacteria shed in the feces of mice orally

201 inoculated with EcN or $\mathrm{PROT}_{3} \mathrm{EcT}-4$ (Fig. 5a). After the administration of a single dose of $\sim 10^{8}$

202 colony forming units (CFU) via oral gavage, EcN and PROT ${ }_{3} \mathrm{EcT}-4$ were each detected in the

203 shed feces at $\sim 10^{5} \mathrm{CFU} / \mathrm{gm} /$ day for at least 14 days, with no significant decrease in fecal

204 shedding over that period (Fig. 5b). Each of the $158 \mathrm{Nb}$-secreting PROT 3 EcT-4 colonies isolated

205 from a total of 4 mice at 2-, 5-, and 14-days post-inoculation secreted Nbs, indicating that their

206 T3SA remained fully functional and that the alr-Nb-expressing plasmid was maintained (Fig. 5c

207 and Fig. S4a-c). No significant weight loss was observed over the course of these experiments 208 (Fig. S4d).

Next, we examined the biogeography of $\mathrm{PROT}_{3} \mathrm{EcT}-3$ and $\mathrm{EcN}$ within the intestines of

210 mice inoculated with variants of each that constitutively express the luciferase-producing

211 luxCDABE operon ${ }^{36}$. The two strains exhibited equivalent luciferase activity when grown in vitro

212 (Fig. S5a-b). Eight days post-oral inoculation of the mice, each strain exhibited similar patterns

213 of luciferase expression in explanted sections of their ileum, cecum, and proximal colon (Fig.

$2145 \mathrm{~d}$ ). In complementary studies, CFUs of $\mathrm{PROT}_{3} \mathrm{ECT}-3$ and $\mathrm{ECN}$ found in various regions of the

215 intestines of orally inoculated mice were equivalent and exceeded $5 \times 10^{9} \mathrm{CFU} / \mathrm{g}$ of contents

216 (Fig. S5c).

To confirm that the modified T3SS present in $\mathrm{PROT}_{3} \mathrm{ECT}$ is actively transcribed within

218 the intestines of mice, we developed a luxCDABE-based reporter that is only activated when the

219 Mxi-Spa operons, which encode the modified T3SA, are transcribed (Fig. S5d). Variants of

$220 \mathrm{PROT}_{3} \mathrm{EcT}-3$, but not EcN, that contained this reporter demonstrated luciferase production in

221 vitro (Fig. S5e-f) and within the explanted cecum, proximal colon, and ileum of inoculated mice

222 (Fig. 5e). These observations establish that the T3SS present within $\mathrm{PROT}_{3} \mathrm{EcT}-3$ is expressed

223 within the intestines of mice and does not interfere with EcN colonization. 
To establish the use of $\mathrm{PROT}_{3} \mathrm{EcT}$ as a therapeutic platform, we focused efforts on investigating its efficacy in the treatment of inflammatory bowel diseases (IBD). The etiologies of

227 ulcerative colitis and Crohn's disease, collectively termed IBD, are complex and thought to be 228 driven by host genetic, environmental, and microbiota factors. Yet, both diseases exhibit chronic 229 inflammation accompanied by increased levels of pro-inflammatory cytokines ${ }^{37}$. Monoclonal 230 antibodies (mAb) that target the pro-inflammatory cytokine TNFa, e.g., infliximab and 231 adalimumab, are highly efficacious in controlling severe disease and in improving the quality of 232 life of patients with $\mathrm{IBD}^{38}$. However, given the systemic administration of these therapeutics, 233 patients receiving these agents are immunosuppressed and at increased risk of developing life234 threatening infections and lymphoma ${ }^{39}$.

We hypothesized that the targeted delivery of anti-TNFa Nbs via PROT 3 EcT to the 236 intestines could reduce intestinal inflammation. To investigate this possibility, we first isolated 237 anti-TNFa Nbs from alpacas immunized with recombinant mouse TNFa, including one that 238 binds with high affinity $\left(\mathrm{EC}_{50} 0.1 \mathrm{nM}\right)$ and neutralizes TNFa $\left(\mathrm{IC}_{50} 0.1 \mathrm{nM}\right)$ (Table $\mathrm{S} 1$ and Figure 239 S6). We generated both monomeric and dimeric variants of Nbs engineered with an OspC2 240 secretion sequence. The dimer was secreted much more efficiently than the monomer (Fig. 6a) 241 and $\mathrm{PROT}_{3} \mathrm{EcT}$ secreted dimeric Nbs were as effective as E. coli-purified dimeric Nbs in 242 blocking TNFa-induced death of mouse L929 cells (Fig. 6b).

\section{TNF-PROT ${ }_{3} \mathrm{EcT}$ inhibits the development of disease in a mouse model of colitis.}

244 To investigate the utility of $\mathrm{PROT}_{3} \mathrm{EcT}$ as a live biotherapeutic for the treatment of 245 intestinal inflammation, we interrogated the therapeutic efficacy of TNF-PROT 3 EcT-4 246 (PROT 3 EcT that constitutively secrete the anti-TNF dimeric $\mathrm{Nb}$ ) in the suppression of TNBS 247 (2,4,6-trinitrobenzene sulfonic acid)-induced colitis. In this preclinical model of IBD, a mixture of 
248 TNBS, a hapten, and ethanol, which disrupts the mucosal barrier, is instilled into the colon via 249 rectal administration. TNBS bound to colonic tissue proteins subsequently induces inflammation 250 driven by pro-inflammatory cytokines, including $\mathrm{TNFa}^{40}$. As previously reported ${ }^{41}$, mice treated 251 intraperitoneally with a neutralizing anti-TNF monoclonal antibody (1-day prior and 2- and 4252 days post-administration of TNBS) were protected from weight loss, colon shortening, and 253 histologic evidence of colitis (Fig. S7).

254 To test the therapeutic efficacy of TNF-PROT ${ }_{3} \mathrm{EcT}$, we orally administered $10^{8} \mathrm{CFU}$ of 255 TNF-PROT 3 EcT-4, PROT ${ }_{3}$ EcT-4, or PBS to mice one day before as well as two and four days 256 after they received TNBS (Fig. 6c). Animals that received bacteria shed equivalent levels of $10^{5}$ $25710^{6} \mathrm{CFU} / \mathrm{g}$ of TNF-PROT ${ }_{3} \mathrm{EcT}-4$ and $\mathrm{PROT}_{3} \mathrm{EcT}-4$ in their feces (Fig. 6d). Treatment with TNF$258 \mathrm{PROT}_{3} \mathrm{EcT}-4$ significantly reduced weight loss, blunted colon shortening, and decreased or 259 completely abrogated epithelial injury and inflammation in the mucosa, including less 260 polymorphonuclear and mononuclear cell infiltration (Fig. 6e-h). In contrast, $\mathrm{PROT}_{3}$ EcT-4 did 261 not provide any protection as assessed by each of these metrics, demonstrating that the 262 therapeutic efficacy afforded by TNF-PROT 3 EcT is due to the secreted TNF-neutralizing $\mathrm{Nb}$ and 263 not EcN intrinsic.

Given that enemas are commonly used for drug delivery for patients with IBD, we also 265 investigated the efficacy of intrarectally delivered TNF-PROT 3 EcT-4 in limiting TNBS-induced 266 colitis using the same dosing strategy as described above (Fig. S8a). As with oral delivery, 267 intrarectally delivered TNF-PROT ${ }_{3} \mathrm{EcT}-4$, but not PROT ${ }_{3} \mathrm{EcT}-4$, ameliorated weight loss, colon 268 shortening, and colitis (Fig. S8b-e). Thus, when delivered either orally or intrarectally, TNF$269 \mathrm{PROT}_{3} \mathrm{EcT}-4$ provides protection against TNFa-driven inflammation in the TNBS model of 270 colitis. 
272 colon may be useful if administered repeatedly to patients. To assess whether treatment with

273 similarly engineered $\mathrm{DH} 10 \mathrm{~b}$ E. coli also suppresses colonic inflammation, we developed $\mathrm{T}_{3}$ EcT,

274 a variant of mT3Ec-Mxi-Spa engineered with the chromosomally encoded PJ23119 VirB gene

275 cassette, and a variant of $\mathrm{T}_{3} \mathrm{EcT}$ that secretes $\mathrm{Nb}^{\mathrm{TNF}}, \mathrm{TNF}-\mathrm{T}_{3} \mathrm{EcT}$. After establishing that TNF-

$276 \mathrm{~T}_{3} \mathrm{EcT}$ constitutively secreted $\mathrm{Nb}^{\mathrm{TNF}}$ into its surroundings (Fig. S8f), TNF- $\mathrm{T}_{3} \mathrm{EcT}$ and $\mathrm{T}_{3} \mathrm{EcT}$ were

277 administered orally or intrarectally to mice using the strategy outline above (Fig. S8a). Orally

278 administered TNF- $T_{3}$ EcT provided no protection, likely due to its inability to colonize the

279 intestines as assessed by fecal shedding (Fig. S8b). In contrast, treatment with rectally

280 delivered TNF- $\mathrm{T}_{3} \mathrm{EcT}$, but not $\mathrm{T}_{3} \mathrm{EcT}$, suppressed colitis (Fig. S8-C-e), likely reflecting repeated

281 transient deposition of anti-TNF Nbs in the colon. These observations demonstrate that the Ipa-

282 Mxi secretion-based platform can be extended to additional E. coli strains.

283 To address whether bacterial secreted Nbs are restricted to the gut, we measured $\mathrm{Nb}$

284 levels in the colonic contents, colon tissue homogenates, and serum of mice treated with each

285 strain across each of the TNBS experiments. To measure the anti-TNF Nb, we used a direct

286 ELISA, which is also capable of detecting the anti-TNF mAb. In mice administered the anti-TNF

$287 \mathrm{mAb}$ via an intraperitoneal route, we detected mAb in the serum of $50 \%$ of the mice (Fig. S9a).

288 By contrast, levels of serum anti-TNF Nb were below the level of detection in all mice orally

289 inoculated with TNF-PROT 3 EcT, and only detectable in $20 \%$ of mice treated with TNF-

$290 \mathrm{PROT}_{3} \mathrm{EcT}$ or TNF-T $\mathrm{EcT}_{3}$ via enema. We did not detect evidence of anti-TNF Nbs in the colonic

291 contents or homogenates (Fig. S9b-c).

292 A single dose of TNF-PROT 3 EcT is associated with TNFa suppression and inhibition of 293 intestinal inflammation. 
Given that the TNF-PROT 3 EcT-4 treated mice exhibited minimal weight-loss postadministration of TNBS, we tested whether pretreatment with a single dose is therapeutically efficacious. Two days post-TNBS administration, mice pre-treated with a single oral dose of TNF-PROT 3 EcT-4 (Fig. 6i) exhibited minimal evidence of weight loss, colon shortening, and 298 colitis (Fig. 6k-m). PROT 3 EcT-4 or the vehicle diluent (PBS) had no effect. As before, similar 299 levels of both strains were shed in the feces (Fig. 6j) and all colonies of shed TNF-PROT 3 EcT-4 300 retained the ability to secrete anti-TNF Nb (Fig S7f). For these experiments, mice were 301 sacrificed two days post-administration of TNBS, a time point at which we reproducibly detected 302 elevated proinflammatory cytokine levels within colonic tissue in controls. Significantly lower 303 levels of TNFa and IL-6 were detectable within the colonic tissue of mice pretreated with TNF-

$304 \mathrm{PROT}_{3} \mathrm{EcT}-4$ (Fig. 6n-o), suggesting that the secreted anti-TNFa Nb sequesters its target and 305 reduces IL-6. While others have observed that TNF neutralization or EcN treatment can 306 increase IL-10 production in the gut, we observed equivalent levels of IL-10, regardless of the 307 intervention (Fig. 6p).

\section{Discussion}

309 Here we describe the development of PROT $_{3}$ EcT, E. coli engineered for the in-situ delivery of 310 high specificity protein payloads to sites of disease. Using synthetic biology-based approaches 311 we have engineered both laboratory and non-pathogenic human $E$. coli isolates with a T3SA 312 modified to secrete proteins in a regulated or constitutive manner. PROT 3 EcT engineered with a 313 constitutively active secretion system that is maintained in the absence of antibiotic selection

314 exhibited growth in vitro at rates equivalent to unmodified $\mathrm{EcN}$ and can colonize the intestines of 315 mice for at least 14 days.

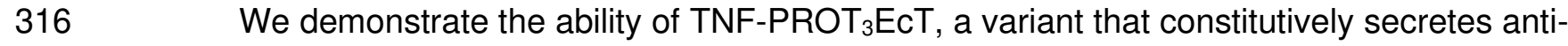
317 TNF Nbs, to suppress the development of inflammation in a preclinical mouse model of IBD. 318 Orally or rectally administered TNF-PROT 3 EcT was as efficacious as systemically administered 
anti-TNF mAb in limiting the development of TNBS-induced colitis. Other groups have also

320 engineered microbes to treat gut inflammation, the most closely related being variants of

321 Lactococcus lactis that secrete IL-10, an anti-inflammatory cytokine, or an anti-TNF Nb ${ }^{42,43}$. A

322 native secretion system of this Gram-positive bacterium was repurposed for the secretion of

323 therapeutic payloads. However, the strain of L. lactis used does not colonize the intestines of

324 humans or mice ${ }^{44,45}$ and may vary in its metabolic activity within the mammalian intestine ${ }^{46}$,

325 likely accounting for why it only moderately suppressed inflammation when administered on a

326 daily basis. By contrast, we observe that pre-treatment with just a single oral dose of TNF-

$327 \mathrm{PROT}_{3} \mathrm{EcT}$ significantly ameliorates colonic inflammation and injury.

The modular design of $\mathrm{PROT}_{3} \mathrm{EcT}$ is such that it can be adapted to secrete different

payloads as well as to respond to environmental cues. For example, in future studies, by

altering the conditions that induce expression of VirB, PROT ${ }_{3} \mathrm{EcT}$ 's T3SA could be endowed

with an 'on switch' triggered by specific signals of the gut's inflammatory milieu, e.g., reactive nitrogen species ${ }^{36,47-49}$. In terms of therapeutic payloads, we demonstrate the versatility of $\mathrm{PROT}_{3} \mathrm{EcT}$ to secrete different Nbs, including $\mathrm{Nbs}$ that inhibit the activity of bacterial toxins

$334\left(\mathrm{Nb}^{\mathrm{St} \times 2}\right)$ or immune checkpoint molecules $\left(\mathrm{Nb}^{\mathrm{PD}-\mathrm{L} 1}\right.$ and $\left.\mathrm{Nb}^{\mathrm{CTLA}-4}\right)$. While the Nbs we studied were each derived from immunized alpacas, synthetic yeast- and bacterial-based Nb libraries are also available that can be screened rapidly for $\mathrm{Nbs}$ with desired properties ${ }^{50,51}$. Furthermore,

$337 \mathrm{PROT}_{3} \mathrm{EcT}$ is not limited to the secretion of Nbs, as we and others have previously established

338 that a variety of other heterologous proteins can be recognized as type III secreted substrates ${ }^{52}$.

339 By altering its route of administration, $\mathrm{PROT}_{3} \mathrm{EcT}$ can be expanded for the deposition of

340 therapeutics not only to the gastrointestinal tract, but also to solid tumors, as EcN home to and

341 colonize a variety of solid tumors when administered intravenously, at least in mice ${ }^{53}$.

342 Given its inherent anti-inflammatory and anti-microbial properties, EcN is GRAS and has

343 been used for over a century to treat various intestinal diseases and is available over-the-

344 counter in some countries. However, EcN contains an operon that mediates the synthesis of a 
colibactin, a genotoxin capable of mediating the formation of DNA crosslinks ${ }^{54}$. Other colibactin-

346 producing E. coli promote the development of colorectal cancer (CRC) in mouse models ${ }^{55}$ and

347 induce mutational signatures found in human $\mathrm{CRC}^{56}$. Whether this will turn out to be an issue

348 that limits the use of EcN-based therapeutics in humans remains to be discovered. However,

349 EcN mutants deficient in colibactin biosynthesis are not impaired in their ability to colonize the

350 intestines of at least mice ${ }^{57,58}$. In future studies, we intend to test the ability of colibactin-

351 deficient EcN-based TNF-PROT ${ }_{3}$ EcT to suppress intestinal inflammation. Herein, we found that

352 two colibactin-negative strains, E. coli $\mathrm{HS}$ and $\mathrm{DH} 10 \mathrm{~B}^{59}$, can also be engineered with a

353 functional secretion system. While the anti-TNF Nb-secreting, E. coli DH10ß based,

354 mT3Ec_Mxi-Spa, was unable to colonize the intestines, rectally administered mT3Ec_Mxi-Spa

355 suppressed TNBS-induced inflammation as efficacious as EcN-based TNF-PROT 3 ECT.

In summary, we describe the development and characterization of $\mathrm{PROT}_{3} \mathrm{EcT}$,

357 programmable E. coli engineered for the site-specific delivery of therapeutic payloads to sites of

358 disease. While the presented studies support the further development of $\mathrm{PROT}_{3} \mathrm{EcT}$ for the

359 treatment of IBD, its modularity permits its rapid adaptation into a therapeutic platform for a

360 broad range of diseases.

\section{References}

1. Sonnenborn, U. \& Schulze, J. The non-pathogenicEscherichia colistrain Nissle 1917 features of a versatile probiotic. Microbial Ecology in Health and Disease 21, 122-158 (2009).

2. Kurtz, C.B. et al. An engineered E. coli Nissle improves hyperammonemia and survival in mice and shows dose-dependent exposure in healthy humans. Sci Transl Med 11, eaau7975 (2019).

3. Isabella, V.M. et al. Development of a synthetic live bacterial therapeutic for the human metabolic disease phenylketonuria. Nat Biotechno/ 36, 857-864 (2018).

4. Crook, N. et al. Adaptive Strategies of the Candidate Probiotic E. coli Nissle in the Mammalian Gut. Cell Host Microbe 25, 499-512 e498 (2019).

5. Danino, T. et al. Programmable probiotics for detection of cancer in urine. Sci Trans/ Med 7, 289ra284 (2015). 
375

376

377

378

379

380

381

382

383

384

385

386

387

388

389

390

391

392

393

394

395

396

397

398

399

400

401

402

403

404

405

406

407

408

409

410

411

412

413

414

415

416

417

418

419

420

421

422

423

6. Din, M.O. et al. Synchronized cycles of bacterial lysis for in vivo delivery. Nature 536, 8185 (2016).

7. Gurbatri, C.R. et al. Engineered probiotics for local tumor delivery of checkpoint blockade nanobodies. Sci Transl Med 12 (2020).

8. Piñero-Lambea, C. et al. Programming Controlled Adhesion of E. coli to Target Surfaces, Cells, and Tumors with Synthetic Adhesins. ACS Synthetic Biology 4, 463-473 (2015).

9. Praveschotinunt, P. et al. Engineered E. coli Nissle 1917 for the delivery of matrixtethered therapeutic domains to the gut. Nat Commun 10, 5580 (2019).

10. Dalbey, R.E. \& Kuhn, A. Protein Traffic in Gram-negative bacteria - how exported and secreted proteins find their way. FEMS Microbiology Reviews 36, 1023-1045 (2012).

11. $\mathrm{Du}$, J. et al. The type III secretion system apparatus determines the intracellular niche of bacterial pathogens. Proc Natl Acad Sci U S A 113, 4794-4799 (2016).

12. Ernst, N.H., Reeves, A.Z., Ramseyer, J.E. \& Lesser, C.F. High-Throughput Screening of Type III Secretion Determinants Reveals a Major Chaperone-Independent Pathway. mBio 9 (2018).

13. Mou, X., Souter, S., Du, J., Reeves, A.Z. \& Lesser, C.F. Synthetic bottom-up approach reveals the complex interplay of Shigella effectors in regulation of epithelial cell death. Proc Natl Acad Sci U S A 115, 6452-6457 (2018).

14. Reeves, A.Z. et al. Engineering Escherichia coli into a protein delivery system for mammalian cells. ACS Synth Biol 4, 644-654 (2015).

15. Buchrieser, C. et al. The virulence plasmid pWR100 and the repertoire of proteins secreted by the type III secretion apparatus of Shigella flexneri. Mol Microbio/ 38, 760771 (2000).

16. Venkatesan, M.M. et al. Complete DNA sequence and analysis of the large virulence plasmid of Shigella flexneri. Infect Immun 69, 3271-3285 (2001).

17. Sasakawa, C. et al. Functional organization and nucleotide sequence of virulence Region-2 on the large virulence plasmid in Shigella flexneri 2a. Molecular Microbiology 3, 1191-1201 (1989).

18. Ménard, R., Sansonetti, P., Parsot, C. \& Vasselon, T. Extracellular association and cytoplasmic partitioning of the IpaB and IpaC invasins of S. flexneri. Cell 79, 515-525 (1994).

19. Ménard, R., Prévost, M.C., Gounon, P., Sansonetti, P. \& Dehio, C. The secreted Ipa complex of Shigella flexneri promotes entry into mammalian cells. Proceedings of the National Academy of Sciences 93, 1254-1258 (1996).

20. Reeves, A.Z. \& Lesser, C.F. Transfer of Large Contiguous DNA Fragments onto a Low Copy Plasmid or into the Bacterial Chromosome. Bio Protoc 6 (2016).

21. Bahrani, F.K., Sansonetti, P.J. \& Parsot, C. Secretion of Ipa proteins by Shigella flexneri: inducer molecules and kinetics of activation. Infect Immun 65, 4005-4010 (1997).

22. Schmidt, F.I. et al. A single domain antibody fragment that recognizes the adaptor ASC defines the role of ASC domains in inflammasome assembly. J Exp Med 213, 771-790 (2016).

23. Ingram, J.R. et al. PD-L1 is an activation-independent marker of brown adipocytes. Nat Commun 8, 647 (2017).

24. Ingram, J.R. et al. Anti-CTLA-4 therapy requires an Fc domain for efficacy. Proc Natl Acad Sci U S A 115, 3912-3917 (2018).

25. Ashour, J. et al. Intracellular expression of camelid single-domain antibodies specific for influenza virus nucleoprotein uncovers distinct features of its nuclear localization. $J$ Virol 89, 2792-2800 (2015). 
424

425

426

427

428

429

430

431

432

433

434

435

436

437

438

439

440

441

442

443

444

445

446

447

448

449

450

451

452

453

454

455

456

457

458

459

460

461

462

463

464

465

466

467

468

469

470

471

472

26. Tremblay, J.M. et al. A single VHH-based toxin-neutralizing agent and an effector antibody protect mice against challenge with Shiga toxins 1 and 2. Infect Immun 81, 4592-4603 (2013).

27. Qian, Z.G., Xia, X.X., Choi, J.H. \& Lee, S.Y. Proteome-based identification of fusion partner for high-level extracellular production of recombinant proteins in Escherichia coli. Biotechnol Bioeng 101, 587-601 (2008).

28. Zhang, G., Brokx, S. \& Weiner, J.H. Extracellular accumulation of recombinant proteins fused to the carrier protein YebF in Escherichia coli. Nature Biotechnology 24, 100-104 (2006).

29. Gao, D., Wang, S., Li, H., Yu, H. \& Qi, Q. Identification of a heterologous cellulase and its $\mathrm{N}$-terminus that can guide recombinant proteins out of Escherichia coli. Microbial Cell Factories 14, 49 (2015).

30. Gao, D., Luan, Y., Liang, Q. \& Qi, Q. Exploring the N-terminal role of a heterologous protein in secreting out of Escherichia coli. Biotechnol Bioeng 113, 2561-2567 (2016).

31. Ulissi, U., Fabbretti, A., Sette, M., Giuliodori, A.M. \& Spurio, R. Time-resolved assembly of a nucleoprotein complex between Shigella flexneri virF promoter and its transcriptional repressor H-NS. Nucleic Acids Res 42, 13039-13050 (2014).

32. Morin, C.E. \& Kaper, J.B. Use of stabilized luciferase-expressing plasmids to examine in vivo-induced promoters in the Vibrio cholerae vaccine strain CVD 103-HgR. FEMS Immunol Med Microbiol 57, 69-79 (2009).

33. Ho, C.L. et al. Engineered commensal microbes for diet-mediated colorectal-cancer chemoprevention. Nat Biomed Eng 2, 27-37 (2018).

34. Hwang, I.Y. et al. Engineered probiotic Escherichia coli can eliminate and prevent Pseudomonas aeruginosa gut infection in animal models. Nat Commun 8, 15028 (2017).

35. Matsumoto, M. et al. Free D-amino acids produced by commensal bacteria in the colonic lumen. Scientific Reports 8, 17915 (2018).

36. Mimee, M. et al. An ingestible bacterial-electronic system to monitor gastrointestinal health. Science 360, 915-918 (2018).

37. Graham, D.B. \& Xavier, R.J. Pathway paradigms revealed from the genetics of inflammatory bowel disease. Nature 578, 527-539 (2020).

38. Neurath, M.F. Current and emerging therapeutic targets for IBD. Nature Reviews Gastroenterology \& Hepatology 14, 269-278 (2017).

39. Sandborn, W.J. \& Loftus, E.V. Balancing the risks and benefits of infliximab in the treatment of inflammatory bowel disease. Gut 53, 780-782 (2004).

40. Wirtz, S. et al. Chemically induced mouse models of acute and chronic intestinal inflammation. Nat Protoc 12, 1295-1309 (2017).

41. Neurath, M.F. et al. Predominant pathogenic role of tumor necrosis factor in experimental colitis in mice. Eur J Immunol 27, 1743-1750 (1997).

42. Steidler, L. et al. Treatment of murine colitis by Lactococcus lactis secreting interleukin10. Science 289, 1352-1355 (2000).

43. Vandenbroucke, K. et al. Orally administered L. lactis secreting an anti-TNF Nanobody demonstrate efficacy in chronic colitis. Mucosal immunology 3, 49-56 (2010).

44. Kimoto, H., Nomura, M., Kobayashi, M., Mizumachi, K. \& Okamoto, T. Survival of lactococci during passage through mouse digestive tract. Can J Microbiol 49, 707-711 (2003).

45. Zhang, C. et al. Ecological robustness of the gut microbiota in response to ingestion of transient food-borne microbes. The ISME Journal 10, 2235-2245 (2016).

46. Drouault, S., Corthier, G., Ehrlich, S.D. \& Renault, P. Survival, physiology, and lysis of Lactococcus lactis in the digestive tract. Appl Environ Microbiol 65, 4881-4886 (1999). 
47. McKay, R. et al. A platform of genetically engineered bacteria as vehicles for localized delivery of therapeutics: Toward applications for Crohn's disease. Bioeng Trans/ Med 3, 209-221 (2018).

48. Riglar, D.T. et al. Engineered bacteria can function in the mammalian gut long-term as live diagnostics of inflammation. Nature Biotechnology 35, 653-658 (2017).

49. Aurand, T.C. \& March, J.C. Development of a synthetic receptor protein for sensing inflammatory mediators interferon- $y$ and tumor necrosis factor- $\alpha$. Biotechnol Bioeng 113, 492-500 (2016).

50. McMahon, C. et al. Yeast surface display platform for rapid discovery of conformationally selective nanobodies. Nature structural \& molecular biology 25, 289-296 (2018).

51. Zimmermann, I. et al. Synthetic single domain antibodies for the conformational trapping of membrane proteins. Elife 7 (2018).

52. Widmaier, D.M. et al. Engineering the Salmonella type III secretion system to export spider silk monomers. Mol Syst Biol 5, 309 (2009).

53. Stritzker, J. et al. Tumor-specific colonization, tissue distribution, and gene induction by probiotic Escherichia coli Nissle 1917 in live mice. Int J Med Microbiol 297, 151-162 (2007).

54. Nougayrède, J.-P. et al. A Toxic Friend: Genotoxic and Mutagenic Activity of the Probiotic Strain Escherichia coli Nissle 1917. mSphere 6, e00624-00621 (2021).

55. Arthur, J.C. et al. Intestinal inflammation targets cancer-inducing activity of the microbiota. Science 338, 120-123 (2012).

56. Pleguezuelos-Manzano, C. et al. Mutational signature in colorectal cancer caused by genotoxic pks(+) E. coli. Nature 580, 269-273 (2020).

57. Olier, M. et al. Genotoxicity of Escherichia coli Nissle 1917 strain cannot be dissociated from its probiotic activity. Gut Microbes 3, 501-509 (2012).

58. Massip, C. et al. Deciphering the interplay between the genotoxic and probiotic activities of Escherichia coli Nissle 1917. PLOS Pathogens 15, e1008029 (2019).

59. Wallenstein, A. et al. ClbR Is the Key Transcriptional Activator of Colibactin Gene Expression in Escherichia coli. mSphere 5, e00591-00520 (2020).

502 


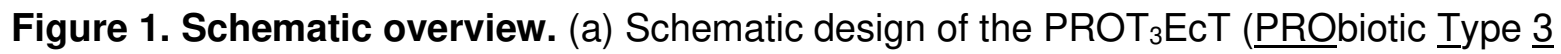
secretion $\underline{E}$. coli Therapeutic) platform, in which the bacteria chassis is engineered with a modified type III secretion apparatus (T3SA) and a therapeutic payload. (b) Schematic of

$508 \mathrm{PROT}_{3} \mathrm{EcT}$ delivering its therapeutic payload directly to the gut lumen.

Figure 2. E. coli engineered with a modified T3SA efficiently secrete proteins into their surroundings. Secretion of FLAG-tagged OspC2 by the indicated strains was monitored by

511 liquid secretion assays sampling at 30-minutes (a, b, e), 6 hours (c) or over a time course at the 512 indicated times (f). (a, b, e, f) Immunoblots of FLAG-tagged OspC2 and GroEL. (c) Coomassie

513 blue stained gel. For each liquid secretion assay, except for (f), the supernatants and pellets

514 were normalized to the lowest $\mathrm{OD}_{600}$. In (f), samples were loaded without normalizing for the

$515 \mathrm{OD}_{600 .}$. In each western blot panel, the images shown are from the same exposure of

516 membranes immunoblotted with designated antibodies. (d) Schematic of PROT 3 EcT-1

517 transformed with pNG-virB and pDSW206-OspC2-FLAG plasmids treated with IPTG. (g) The

518 ability of strains to invade intestinal epithelial cells (HCT8) was determined by gentamicin

519 protection assays. Each data point represents a single bacterial culture. Means are marked with

520 horizontal lines. Data were analyzed using one-way ANOVA with Tukey's post-hoc test; for all 521 strain comparisons to Shigella ${ }^{* *} \mathrm{P}<0.0001$; ns $=$ not significant (both $\mathrm{P}>0.999$ ). Data in each 522 panel is representative of results from at least 2 independent experiments. $C R=C$ Congo red. $P=$ 523 whole cell pellet lysates, $\mathrm{S}=$ supernatant fractions.

525 Figure 3. $\mathrm{PROT}_{3} \mathrm{EcT}$ can be engineered to secrete nanobodies. $6 \mathrm{~h}$ liquid secretion assays 526 monitoring the secretion of (a) $\mathrm{HA}$-tagged $\mathrm{Nb}^{\mathrm{ASC}}$ with different $\mathrm{N}$-terminal type III secretion 527 signals, (b) HA-tagged-Nb ${ }^{A S C},-\mathrm{Nb}^{\mathrm{PD}-\mathrm{L1} 1},-\mathrm{Nb}^{\mathrm{CTLA}-4}$ and $-\mathrm{Nb}^{\mathrm{NP1} 1}$ with an N-terminal OspC2 secretion 
528 signal (OspC2ss), (c) FLAG-tagged $\mathrm{Nb}^{\text {Stx2 }}$ monomer (1x), dimer (2x) and trimer (3x) with $\mathrm{N}$ -

529 terminal OspC2ss and (d) FLAG-tagged $\mathrm{Nb}^{\mathrm{St} \times 2}$ dimer modified with an N-terminal OspC2ss

$530\left(\right.$ OspC2ss- $\left.\mathrm{Nb}^{2 x}\right)$. Data in each panel is representative of results from at least 2 independent

531 experiments. $\mathrm{P}=$ whole cell pellet lysates, $\mathrm{S}=$ supernatant fractions

533 Figure 4. Development of nanobody-secreting constitutively active $\mathrm{PROT}_{3} \mathrm{EcT}$. $2 \times \mathrm{Nb}^{\mathrm{St} 22}$

534 secretion was monitored for $18 \mathrm{~h}$ to compare (a) the performance of different virB promoters in $535 \mathrm{PROT}_{3} \mathrm{EcT}-1$, (c) plasmid encoded (PL) versus chromosomally integrated (INT) virB under the

536 control of $\mathrm{P}_{\mathrm{J} 23119}$ in $\mathrm{PROT}_{3} \mathrm{EcT}_{-1}$ and (d) Ptac ( $\left.\mathrm{P}_{\mathrm{IPTG}}\right)$ versus $\mathrm{P}_{\mathrm{J} 23108}$ driven $2 \times \mathrm{Nb}^{\text {Stx2 }}$

537 expression/secretion in $\mathrm{PROT}_{3} \mathrm{EcT}-3$. (b) Schematic of PROT3EcT-3 with virB under a 538 constitutive promoter $(\mathrm{Pc})$ integrated and pDSW206-OspC2-2xNb ${ }^{\mathrm{St} \times 2}$. (e) Schematic of

$539 \mathrm{PROT}_{3}$ EcT-4 with pCPG-alr-PJ23108-OspC2-2xNb ${ }^{\mathrm{St} \times 2}$. (f) $2 \times \mathrm{Nb}^{\mathrm{St} \times 2}$ expression and secretion in

$540 \mathrm{PROT}_{3} \mathrm{EcT}-3$ and $\mathrm{PROT}_{3} \mathrm{EcT}-4$ grown in the presence or absence of ampicillin (Amp); secretion

541 was monitored for $18 \mathrm{~h}$. (g) Growth rate of strains in LB media without antibiotics. Data are

542 presented as the mean \pm SD and are representative of results from at least 2 independent

543 experiments. Data were analyzed using two-way ANOVA with Tukey's post-hoc test. ns $=$ not

544 significant compared to $\mathrm{EcN}\left(\mathrm{PROT}_{3} \mathrm{EcT} 3, \mathrm{P}=0.7622 ; \mathrm{PROT}_{3} \mathrm{EcT} 4\right.$ + pCGP-alr, $\mathrm{P}=0.9612$;

$545 \mathrm{PROT}_{3} \mathrm{EcT}-4$ + pCGP-alr-PJ23108-OspC2-2xNb $\left.{ }^{\mathrm{S} \times 2}, \mathrm{P}=0.5957\right)$

547 Figure 5. $\mathrm{PROT}_{3} \mathrm{EcT}$ stably colonizes the gastrointestinal tract of mice. (a) Study design.

548 C57/BL6 mice were orally gavaged with $10^{8} \mathrm{CFU}$ of EcN or PROT ${ }_{3} \mathrm{EcT}-4$ and fecal pellets were

549 sampled at the times indicated. (b) Shed bacterial titers as measured by plating homogenates of

550 fecal pellets on selective media and enumerating colonies. Data are presented as the mean \pm

551 SEM, $\mathrm{n}=4$ mice per group and represent at least 2 independent experiments. Data were 
552 analyzed using two-way ANOVA with Tukey's post hoc test. ns $=$ not significant $(P=0.4846)$. (c)

$5536 \mathrm{~h}$ plate secretion assay of colonies of PROT3EcT-4 shed from mice at $14 \mathrm{dpi}$. Membranes are

554 removed and probed with an anti-FLAG to monitor $\mathrm{Nb}^{\mathrm{TNF}}$ secretion. (d-e) Bioluminescent

555 imaging of intestinal explants from individual mice inoculated with strains expressing a

556 constitutive bioluminescent reporter pMM543 (d) or pMxiE-lux++pNG162-IpgC (e) at 8 dpi. Dpi

557 = days post inoculation.

559 Figure 6. TNF-PROT ${ }_{3} \mathrm{EcT}$ inhibits the development of disease in a mouse model of colitis.

560 (a) $6 \mathrm{~h}$ liquid secretion assays monitoring the secretion of FLAG-tagged $\mathrm{Nb}^{\mathrm{TNF}}$ monomer (1x)

561 and homodimer (2x) fused to the $\mathrm{N}$-terminal OspC2ss by $\mathrm{PROT}_{3} \mathrm{EcT}-1 . \mathrm{P}=$ whole cell lysate, $\mathrm{S}$

$562=$ precipitated supernatant. (b) Viability of L929 cells following incubation with $0.2 \mathrm{ng} / \mathrm{ml}$ of

563 murine TNFa plus supernatants from PROT3EcT-1 induced to secrete the $\mathrm{Nb}^{\mathrm{TNF}}$ dimer,

$564 \mathrm{PROT}_{3} \mathrm{EcT}-1$ with empty vector and purified $\mathrm{Nb}^{\mathrm{TNF}}$ dimer. Sup = supernatant. Data were

565 analyzed using a two-way ANOVA with Tukey's post hoc test ns $=$ not significant $(P=0.9980)$ (c)

566 Study design. BALB/c mice received TNBS (2 mg, enema in 50\% ethanol) plus oral gavages of

567 PBS $(n=10)$ or an inoculum of $10^{8}$ CFU of PROT 3 EcT-4 $(n=10)$ or TNF-PROT ${ }_{3}$ EcT-4 $(n=9)$ at

568 the times indicated and were sacrificed at 5 days post TNBS. (d) Shed bacteria. $P=0.3230$. (e)

569 Body weight change (\%). "denotes comparison to PBS group, $\mathrm{P}=0.0118$; " denotes comparison

570 to $\mathrm{PROT}_{3} \mathrm{EcT}-4$; day $1, \mathrm{P}=0.0238$; day 2, $\mathrm{P}=0.0122$. (f) Colon length. *, $\mathrm{P}=0.0219$; ***,

$571 \mathrm{P}=0.0004$. (g) Histologic colitis scores. top *, $\mathrm{P}=0.0231$; bottom * $\mathrm{P}=0.0141$. (h) Representative

572 histology of colon sections stained with hematoxylin and eosin from each experimental group. (i)

573 Study design. Mice were treated with TNBS plus oral gavages of PBS $(n=10)$ or an inoculum of

$57410^{8} \mathrm{CFU}$ of PROT ${ }_{3}$ EcT-4 $(n=9)$ or TNF-PROT 3 EcT-4 $(n=10)$ and sacrificed at 2 days post TNBS.

575 An additional group of mice treated with ethanol alone was included $(n=5)$. (j) Shed bacteria.

$576 \mathrm{P}=0.1758$. (k) Body weight change (\%). *denotes comparison to PBS, day $1, \mathrm{P}=0.0002$, day 2 , 
$577 \mathrm{P}<0.0001$; \#denotes comparison to $\mathrm{PROT} 3 \mathrm{EcT}-4$, day $1, \mathrm{P}=0.054$, day $2, \mathrm{P}<0.0001$. (I) Colon

578 length. *, $\mathrm{P}=0.0184 ;{ }^{* *}, \mathrm{P}=0.0029$. (m) Histologic colitis scores. **, $\mathrm{P}=0.0045$. Colon

579 homogenates were analyzed for the levels of TNFa $(n)\left(^{* * *}, P=0.0005,{ }^{*}, P=0.0433\right), I L-6(0)$

580 (right *,$P=0.0356$; left *,$P=0.0322$ ) and IL-10 ( $p)$ by ELISA. ( $d-g, j-p)$ Data were combined from

5812 independent experiments and are presented as individual values \pm SEM ( $\mathrm{f}-\mathrm{g}, \mathrm{I}-\mathrm{p})$ or mean \pm

582 SEM (d-e, j-k). Data were analyzed using a Kruskal-Wallis test with Dunn's multiple correction

583 test (f, g, I-p) or a two-way ANOVA with Tukey's post hoc test (j, k). TNBS = 2,4,6-

584 Trinitrobenzenesulfonic acid. $\mathrm{EtOH}=$ ethanol.

\section{Acknowledgements}

587 The authors thank Pam Silver for critically reading the manuscript. Drs. Gökhan S. Hotamışlıgil

588 and Karen Inouye at the Harvard T. H. Chan School of Public Health for their assistance with

589 the IVIS experiments and Sue Chapman for assistance in the L929/TNF assay. Some graphics

590 were created with BioRender.com. Supported by NIH grants (AI064285, DK113599), a Kenneth

591 Rainin Foundation grant and the Brit d'Arbeloff Research Scholar award to C.F.L,

592 Massachusetts General Hospital Executive Committee on Research Fund for Medical Discovery

593 Postdoctoral Fellowship Awards to C.G.P and A.Z.R., and an Endeavour Australia Research

594 Fellowship and a Crohn's \& Colitis Foundation Research Fellow Award, award number 654758,

595 to J.P.L. The authors thank members of the Lesser, Garrett, Leong and Goldberg labs for

596 helpful discussions and suggestions.

597

\section{$598 \quad$ Author Contributions}

599 J.P.L., C.G.P., A.Z.R., J.M.L., C.B.S., W.S.G., and C.F.L designed experiments, interpreted 600 data. J.P.L., C.G.P., A.Z.R., N.S., J.M.T and U.P. performed experiments and analyzed data. 
601 F.I.S and H.L.P generated and provided plasmids. J.N.G performed the histology scoring. J.P.L 602 and C.F.L wrote the manuscript. C.G.P., A.Z.R., F.I.S, J.M.L., C.B.S., and W.S.G., edited the 603 manuscript.

604

605 Competing Interests statement

606 The authors declare no competing financial interests. Related to this work, C.F.L is on the 607 scientific advisory board (SAB) of Synlogic Therapeutics and W.S.G is on the SABs of Kintai 608 Therapeutics, SanaRx, Evelo Biosciences and Tenza. F.I.S. is a consultant and shareholder of 609 IFM Therapeutics and NewCo (to be changed if public), as well as cofounders and shareholders 610 of Dioscure Therapeutics SE. 
Figure 1

a

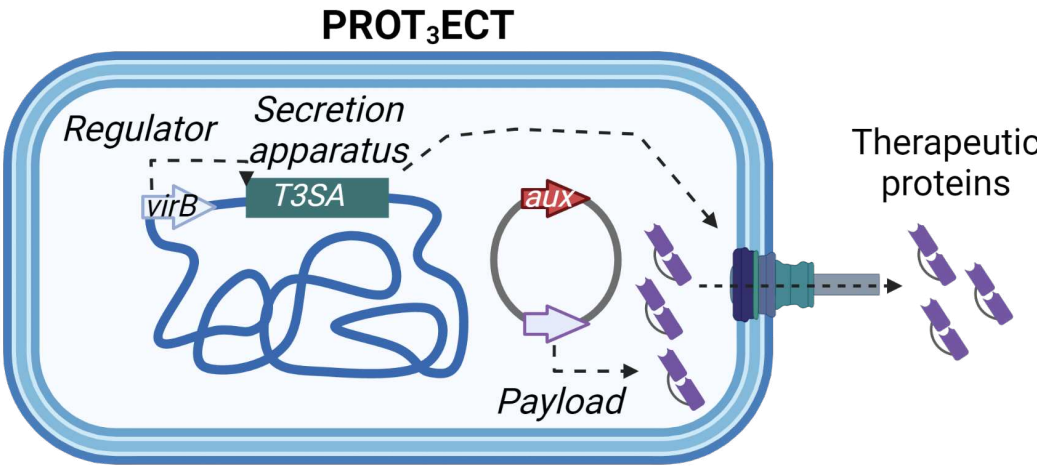

b

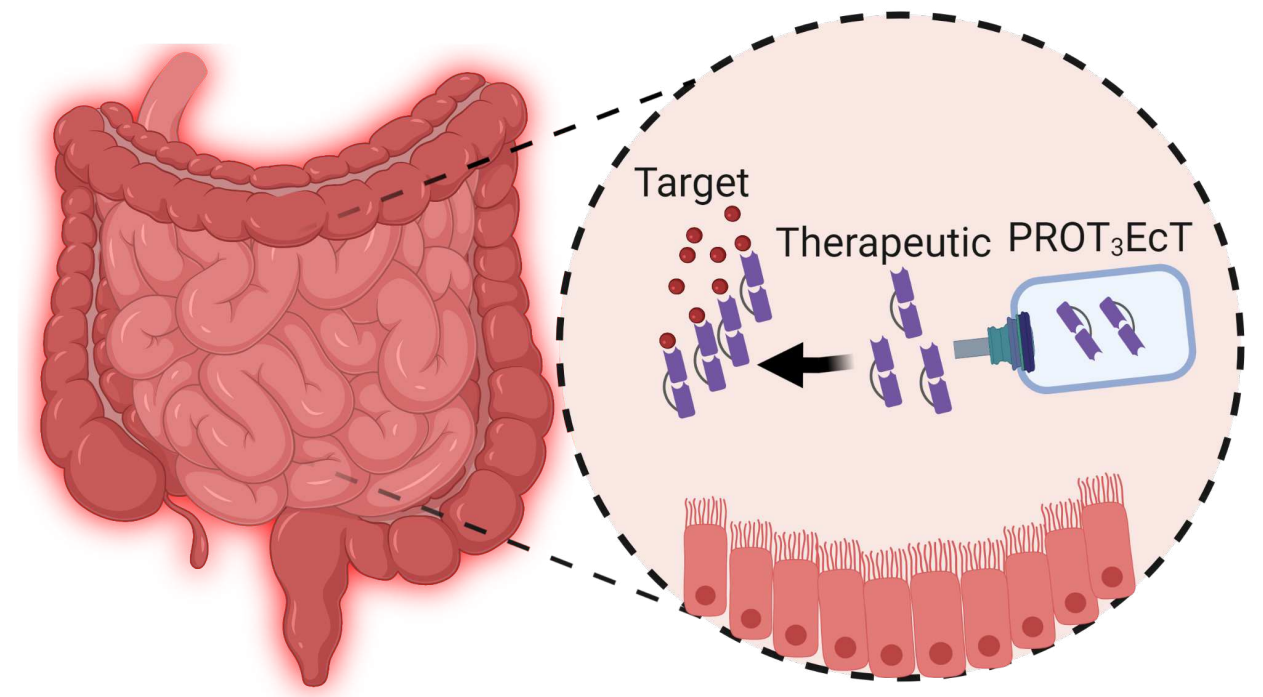


Figure 2

a
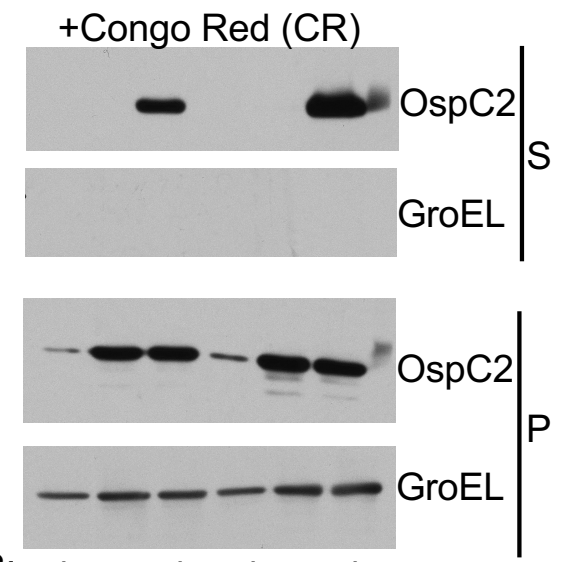

VirB:

IPTG: b
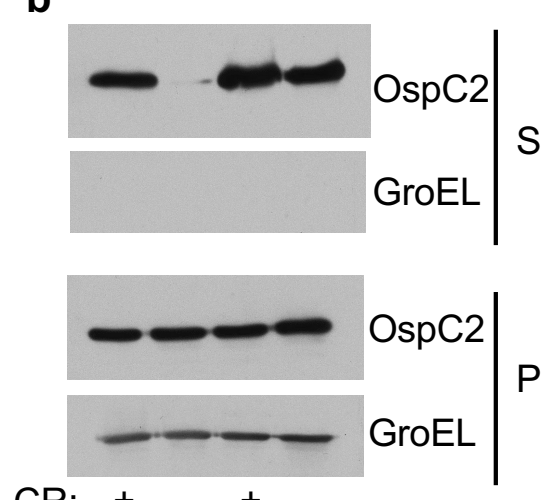

Ipa-Mxi-Spa $\frac{+\quad-}{\text { Mxi-Spa }}$
C

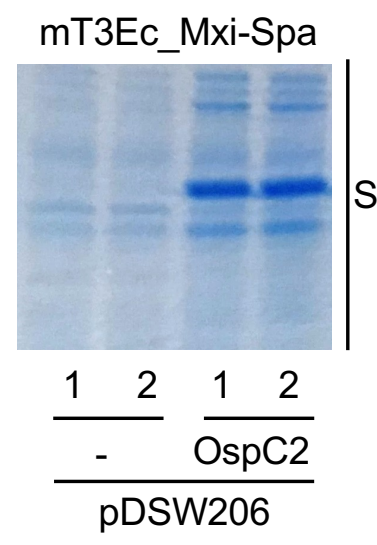

e

d
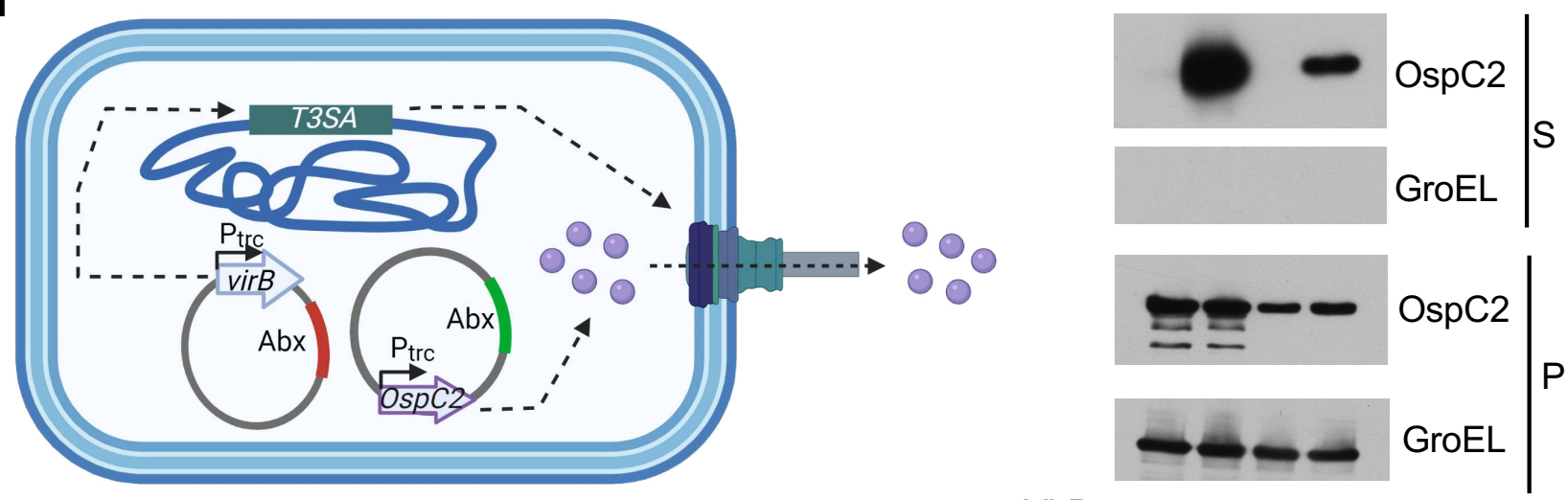

VirB:

mT3Ec_Mxi-Spa PROT $_{3}$ EcT-1

f

mT3Ec-Mxi-Spa PROT $_{3}$ EcT-1

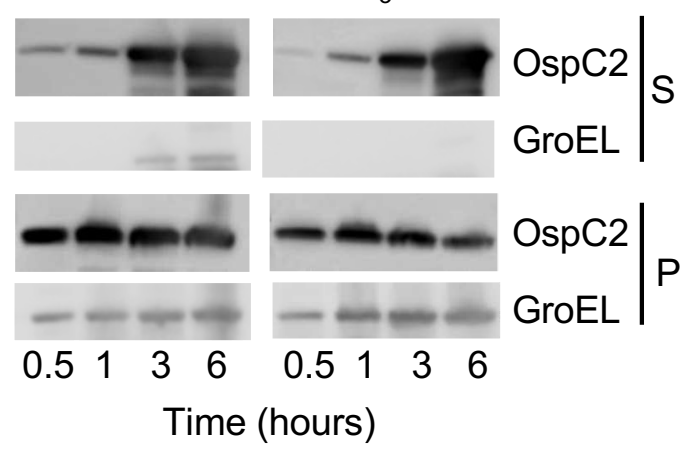

g

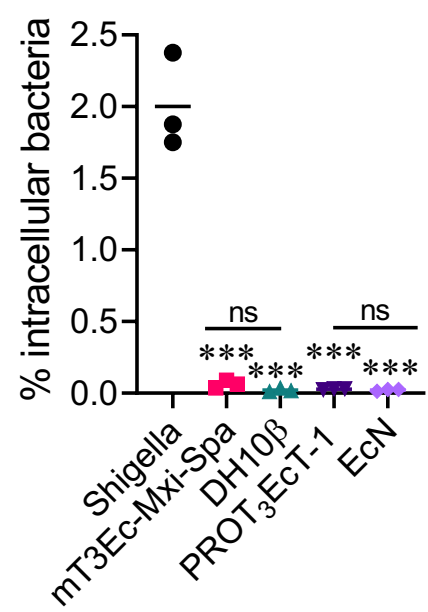


Figure 3

a
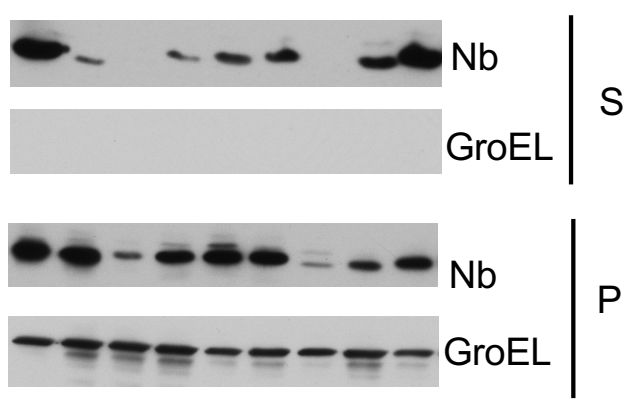

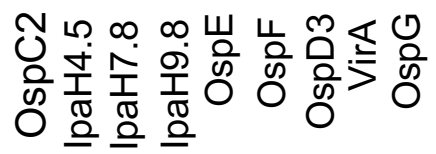

Type III secretion signal (ss)

C
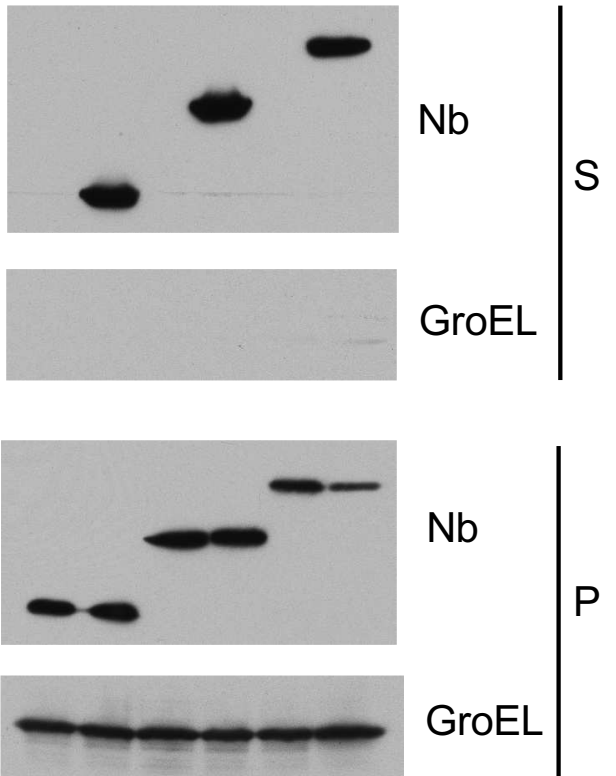

b
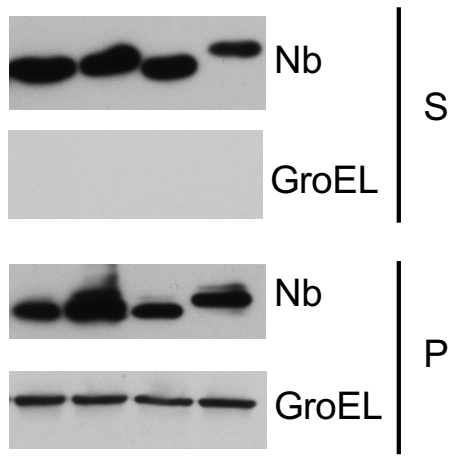

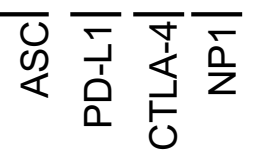

OspC2ss-Nb

d

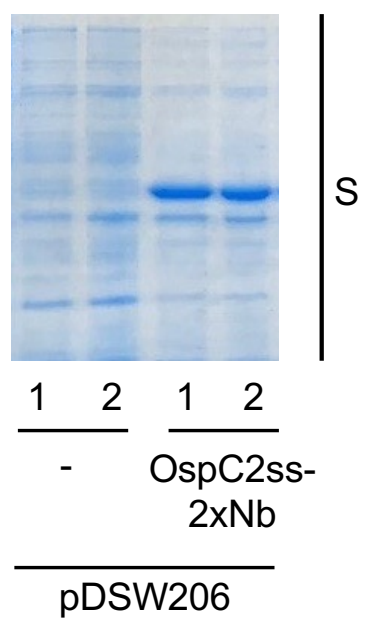

virB:

$-+-+-+$

$\mathrm{Nb}: \frac{1 \mathrm{x}}{2 \mathrm{x}} \frac{-}{3 \mathrm{x}}$ 
Figure 4

a

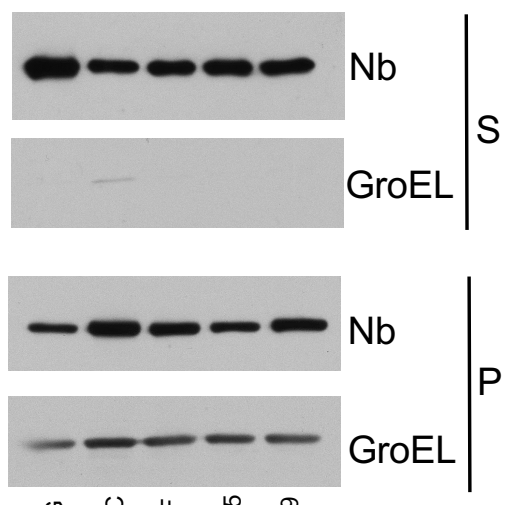

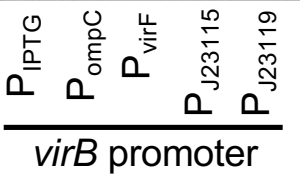

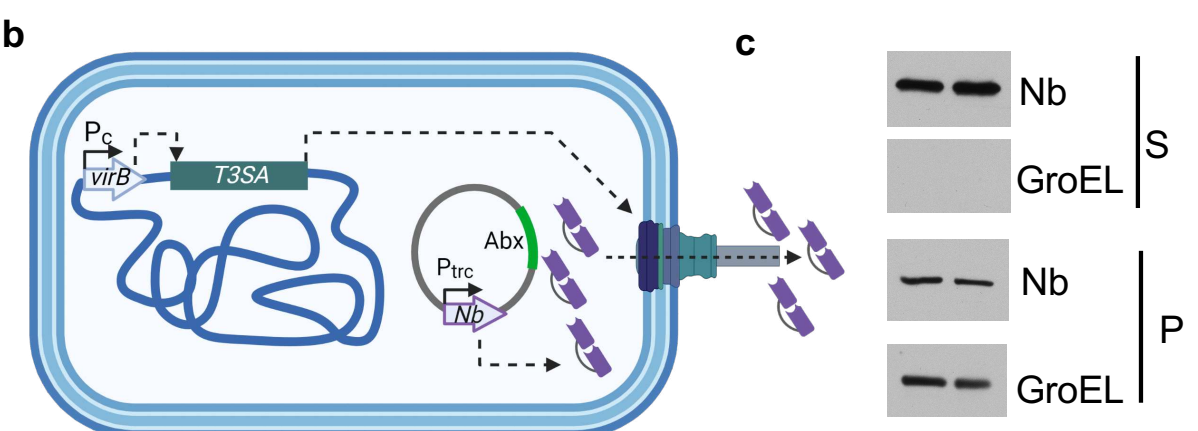

d
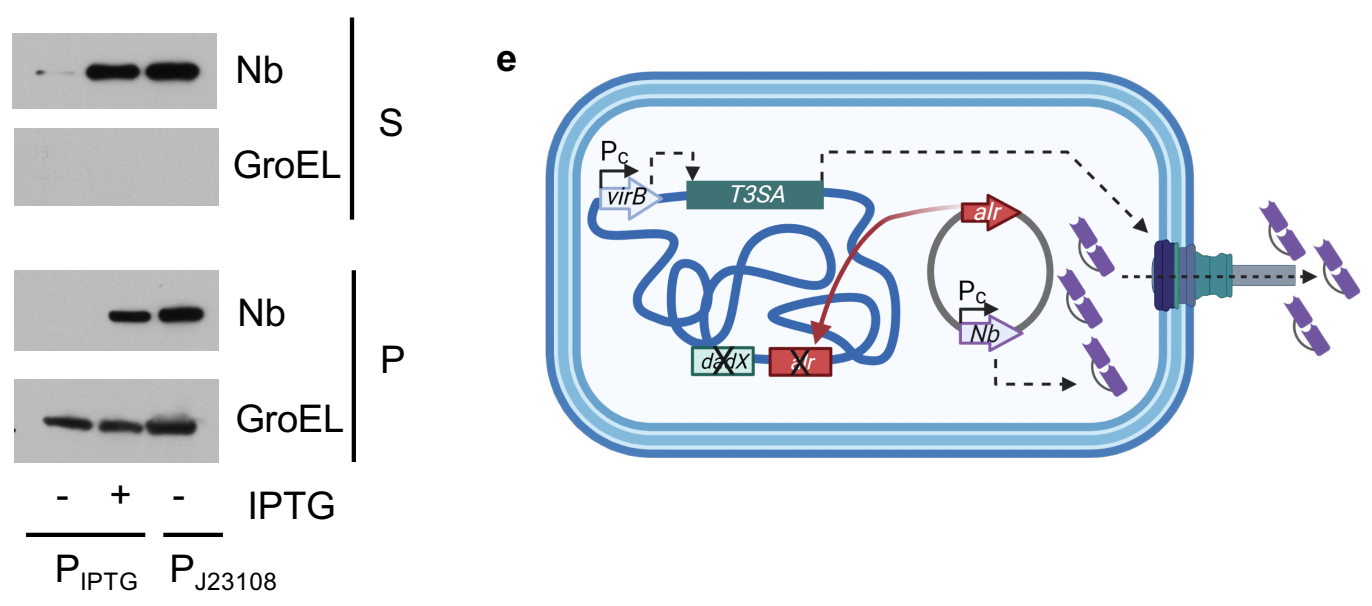

$\mathrm{PROT}_{3}$ ECT-3

f

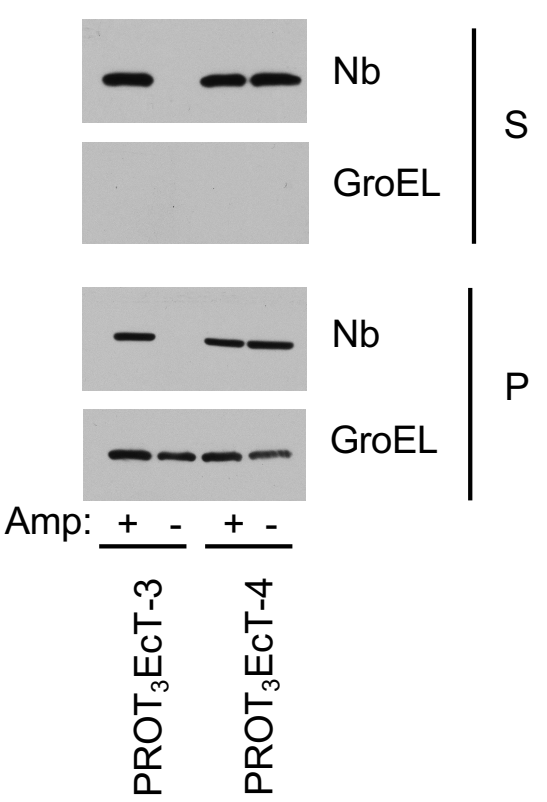

g

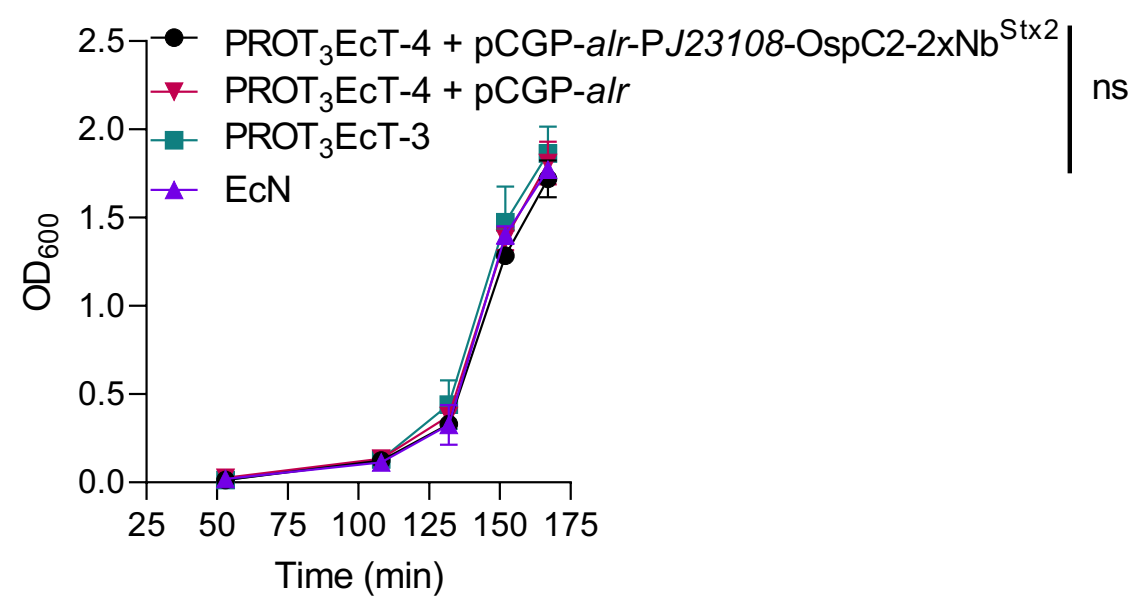


Figure 5

a

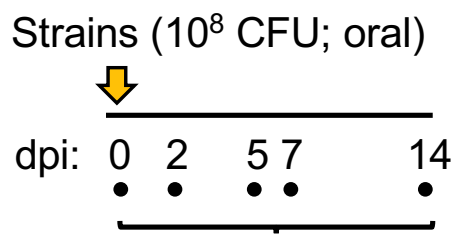

Fecal sampling b

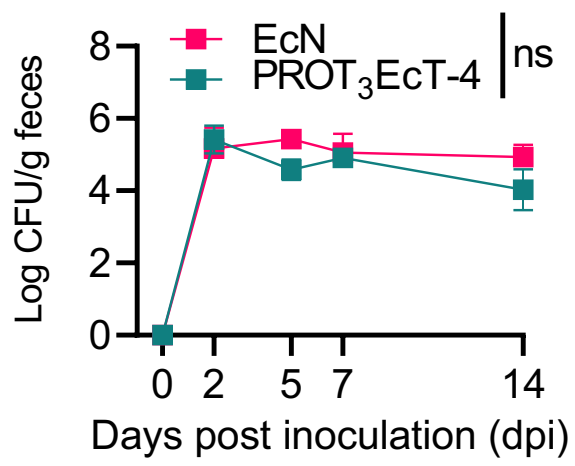

C Colony

\begin{tabular}{|c|c|c|c|c|c|c|c|c|c|c|c|c|c|c|c|}
\hline $14 d p i$ & 1 & 2 & 3 & & 4 & 5 & 6 & & 7 & & 8 & 9 & 10 & 1 & 2 \\
\hline \multirow{4}{*}{ 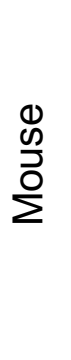 } & & $\bullet$ & $\bullet$ & $\bullet$ & & $\bullet$ & : & & $\bullet$ & & $\bullet$ & : & $\begin{array}{ll}\bullet & \\
\bullet & \bullet\end{array}$ & $\begin{array}{lll} & \bullet & \bullet \\
\bullet & \bullet\end{array}$ & $\bullet \bullet$ \\
\hline & & $\bullet$ & $\bullet$ & $\bullet$ & $\bullet$ & $\bullet$ & $\bullet$ & 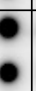 & $\bullet$ & & 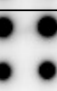 & 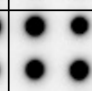 & $\begin{array}{ll}\bullet & \bullet \\
\bullet & \bullet\end{array}$ & \multirow{3}{*}{\multicolumn{2}{|c|}{ Inoculum }} \\
\hline & & $\bullet$ & 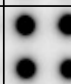 & 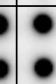 & $\bullet$ & & $\bullet$ & $\bullet$ & $\bullet$ & & $\cdot \bullet$ & 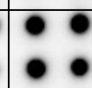 & 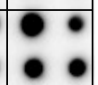 & & \\
\hline & $\begin{array}{ll}0 & 0 \\
0 & 0\end{array}$ & $\bullet$ & 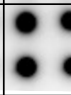 & & $\bullet$ & & $\because$ & $\bullet$ & $\because$ & & 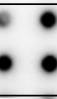 & $0^{\circ}$ & $\begin{array}{ll}1 & \ddots \\
\bullet & \bullet\end{array}$ & & \\
\hline
\end{tabular}

d

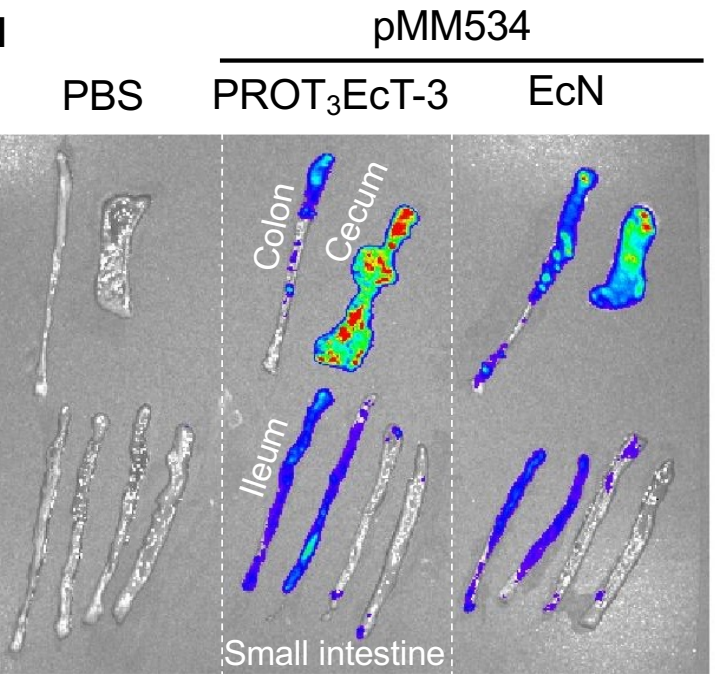

e pMxiE-lux+ +pNG162-lpgC
PBS

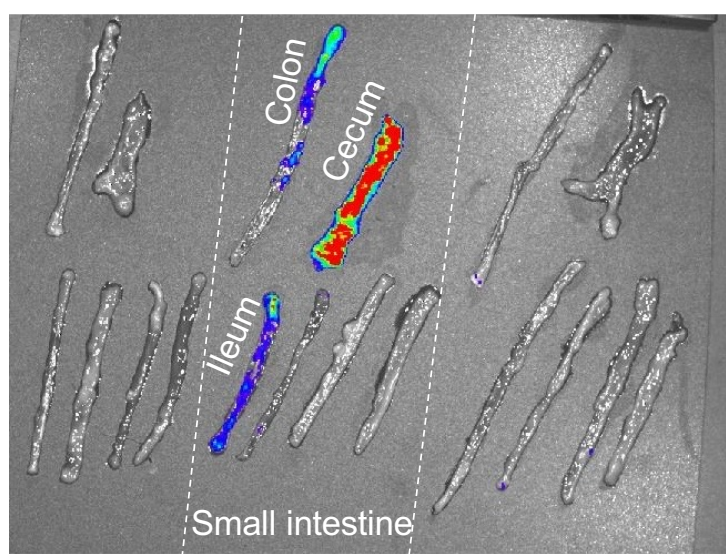




\section{Figure 6}

a

C

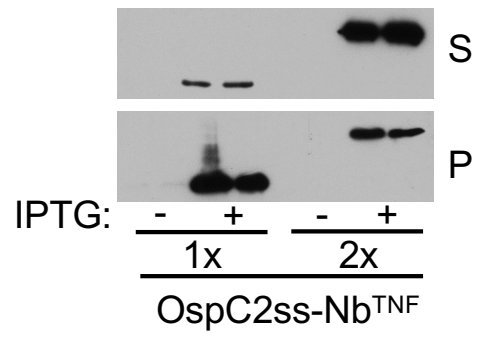

Strains (oral gavage, $10^{8} \mathrm{CFU}$ ) $\checkmark$ TNBS (enema, 2 mg; $50 \%$ EtOH) b

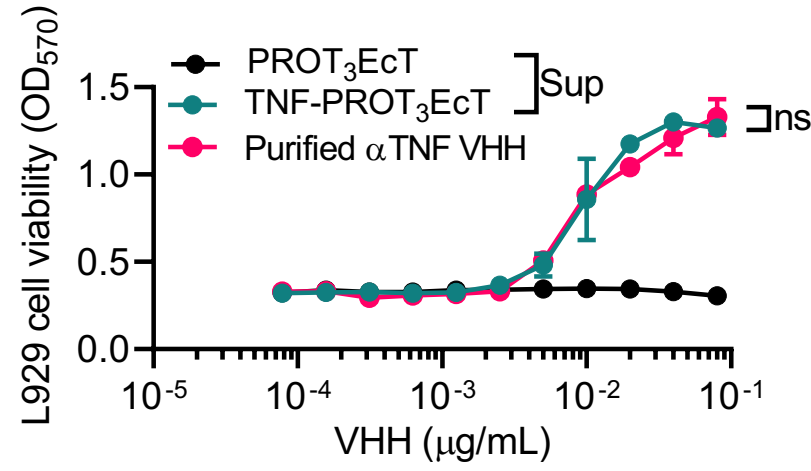

d

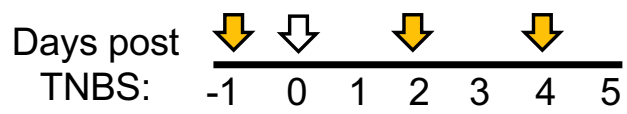

Treatment:

$\rightarrow$ PBS

$\rightarrow \mathrm{PROT}_{3} \mathrm{EcT}_{-4}$

$\rightarrow$ TNF-PROT 3 EcT-4

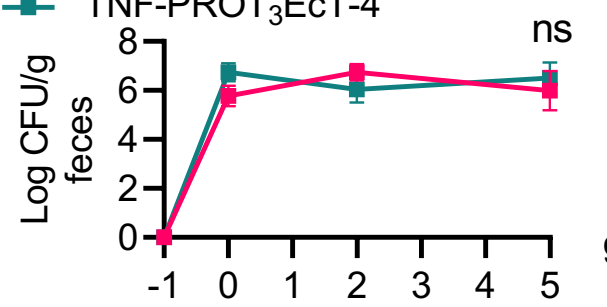

Days post TNBS

e

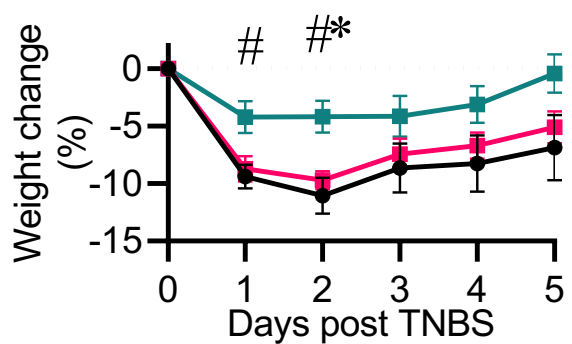

f

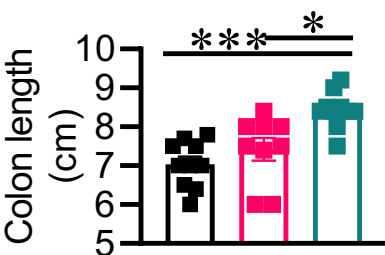

g

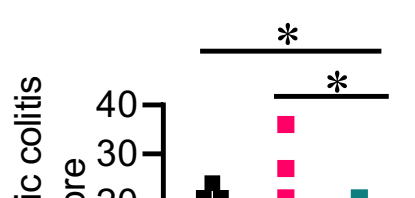

O $030-$

$\frac{0}{\text { 음 }}$

Strains (oral gavage, $10^{8} \mathrm{CFU}$ )

\TNBS (enema, 2 mg; 50\% EtOH) h
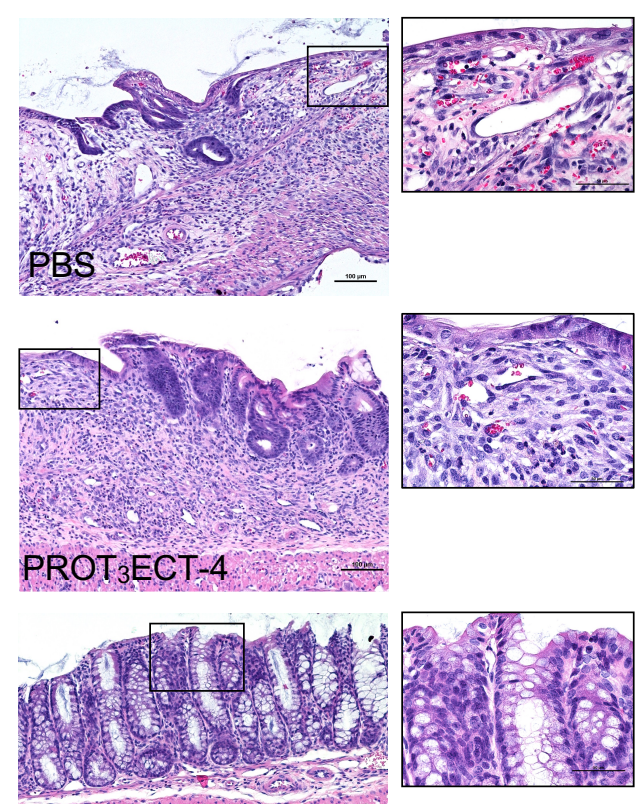

TNF-

$\mathrm{PROT}_{3}$ ECT 4
PROT 3 ECT-4 j

$-\infty \mathrm{EtOH}$

$\rightarrow \mathrm{EtOH}+$ TNBS + PBS

$\rightarrow \mathrm{EtOH}+\mathrm{TNBS}+\mathrm{PROT}_{3} \mathrm{EcT}-4$

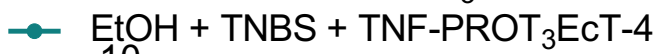

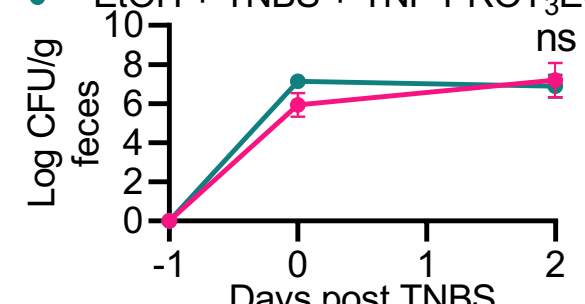

k

Days post TNBS

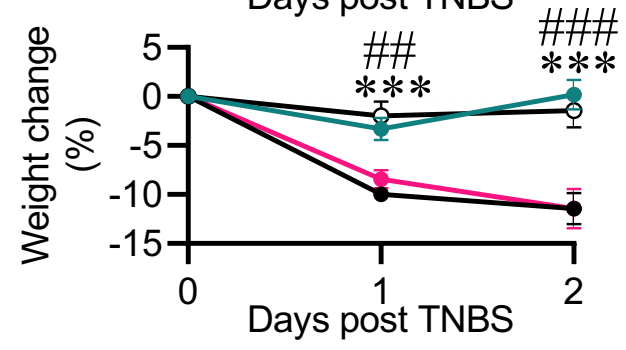

I

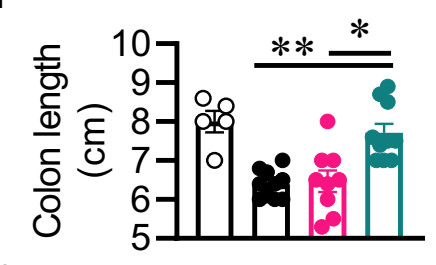

n

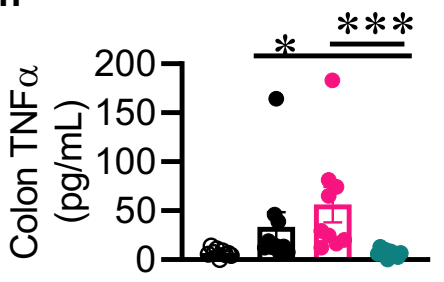

p

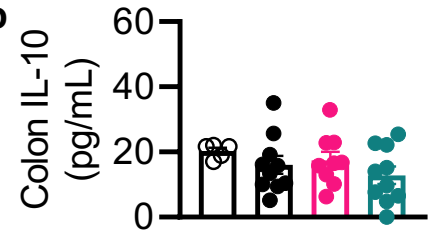

m
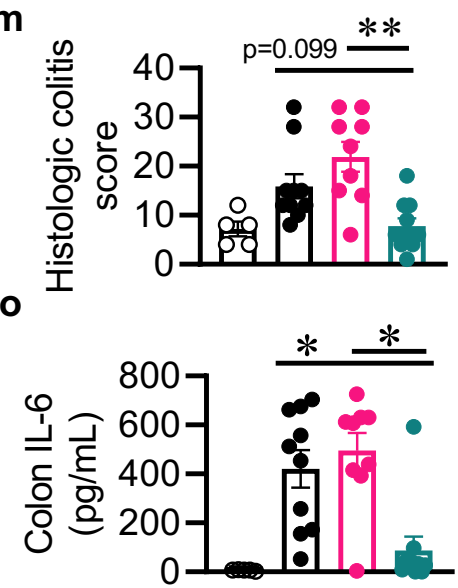


\section{Methods}

2 Plasmids, bacterial strains, cell lines and mouse strains.

3 Plasmids and strains are summarized in Supplementary Tables 2 and 3, respectively.

4 Sequences of oligos and DNA inserts are summarized in Supplementary Tables 4 and 5,

5 respectively. All gateway entry clone inserts were sequence verified. Synthetic DNA fragments

6 were purchased from Integrated DNA technologists (IDT) or Genewiz. All restriction enzymes

7 purchased from New England Biolabs. L929 fibroblast cells and HCT8 cells were grown as

8 recommended by the American Type Culture Collection (ATCC). C57BL/6J mice were used for

9 colonization studies and BALB/CJ mice for the TNBS colitis models. All mice were obtained from

10 The Jackson Laboratory and were housed in microisolator cages in the barrier facility of Harvard

11 T.H. Chan School of Public Health. Animal experiments were approved and carried out in

12 accordance with Harvard Medical School's Standing Committee on Animals and the National

13 Institutes of Health guidelines for animal use and care.

\section{Plasmid construction}

$15 \quad N b^{P D-L 1}, N b^{C T L A-4}, N b^{A S C}$ and $N b^{N P 1}$ Gateway compatible destination vectors: Gateway compatible

16 destination vectors that enable the in-frame introduction of sequences upstream of $\mathrm{Nb}^{\mathrm{PD}-\mathrm{L} 1}$,

$17 \mathrm{Nb}^{\mathrm{CTLA}-4}, \mathrm{Nb}^{\mathrm{ASC}}$ and $\mathrm{Nb}^{\mathrm{NP} 1}$ were generated by using Gibson cloning (NEB) to join (1) PCR

18 amplified Nb-HA fragments from pHEN6 VHH52 [anti-IAV NP]/VHH KV 022[anti-IAV NP]/VHH

19 PD-L1 B3/VHH CTLA-4 H11/VHH ASC with oligomers (P1/P2) and (2) pDSW206-ccdB-MyoD

20 NS with oligomers (P3/P4). Each VHH containing fragment was introduced into the pDSW206

21 based vectors via Gibson cloning (NEB). Resulting clones were sequence verified. The resulting

22 plasmids are referred to as pDSW206-ccdB-Nb* ( ${ }^{*}=$ name of $\mathrm{Nb}$ present in construct). 
$23 \quad N b^{P D-L 1}, N b^{C T L A-4}, N b^{A S C}$ and $N b^{N P 1}$ expression plasmids. A variety of type III secretion signal

24 sequences were introduced into pDSW206-ccdB-Nb ${ }^{A S C}$ via LR reaction using pENTR-secretion

25 sequence entry clones. An OspC2 secretion signal (OspC2ss) was introduced into pDSW206-

26 ccdB-Nb ${ }^{P D-L 1}$, pDSW206-ccdB-Nb ${ }^{C T L A 4}$ or pDSW206-ccdB-Nb ${ }^{N P 1}$ via a LR reaction with pENTR-

27 OspC2ss.

28 Alr plasmids. A DNA fragment containing alr and its native promoter with flanking attB1 and 29 attB2 sites PCR-amplified from EcN using oligomers (P5/P6) was introduced into pDONR221

30 via a BP reaction followed by $\mathrm{pCMD136-ccdB-FLAG} \mathrm{via} \mathrm{an} \mathrm{LR} \mathrm{reaction.} \mathrm{The} \mathrm{resulting} \mathrm{plasmid,}$

31 pCMD136-alr, was used as a template for nested PCR with oligomers (P7/P8, P7/P9 and

$32 \mathrm{P} 10 / \mathrm{P} 11)$ to generate a DNA fragment (BspHI-Palr-alr-T7t-Asel) that was introduced via

33 traditional cloning into pDSW206 ${ }^{1}$ digested with Ncol/Ndel to create pCGP-alr.

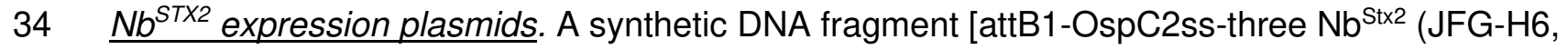
35 JFD-A5 and JGH-G1)-E-tag-attB2] was introduced into pDONR221 via a BP reaction followed by pDSW206-ccdB-3xFLAG via an LR reaction to create pDSW206-OspC2ss-3xNb ${ }^{S t \times 2}$. Dimeric

37 (JFG-H6, JFD-A5) and monomeric (JFG-H6) DNA fragments PCR amplified from pDSW206-

OspC2ss-3xNb ${ }^{\mathrm{Stx} 2}$ using oligomers (P12/P13 and P12/P14) were introduced into pDONR221 via

39 a BP reaction followed by pDSW206-ccdB-3xFLAG via LR reactions to create pDSW206-

40 OspC2ss-2xNbStx2 and pDSW206-OspC2ss-Nb ${ }^{\mathrm{St} \times 2}$.

41 To replace the IPTG-inducible Ptrc promoter and the laclq repressor in pDSW206-OspC2ss-

$422 \mathrm{xNb}^{\mathrm{St} \times 2}$ with a constitutive promoter, two complementary oligos (P15/P16) were annealed to 43 create a BBa_J23115 promoter (Anderson Collection) with cohesive Sphl and Sacl ends. The 44 dsDNA fragment was cloned into Sphl/Sacl digested pDSW206-OspC2ss-2xNb ${ }^{\text {Stx2 }}$ to create 45 pDSW206-J23115-OspC2ss-2xNb ${ }^{\text {Stx2 }}$. The (PJ23115-OspC2ss-2xNb ${ }^{\text {Stx2 }}$ ) fragment in this 46 plasmid was PCR amplified (P17/P18) and introduced into Kpnl/Xbal-digested pCGP-alr to 
create pCGP-alr-PJ23115-OspC2ss-2xNbStx2. Two complementary oligos (P19/P20) were annealed to create a BBa_J23018 (Anderson Collection) promoter with cohesive Sacl ends.

49 The dsDNA fragment was cloned into Sacl-digested pCGP-alr-PJ23115-OspC2ss-2xNb ${ }^{\text {Stx2 }}$ 50 replacing PJ23115 to create pCGP-alr-PJ23108-OspC2ss-2xNbSt×2.

51 OsmY-, N20-Cel-CD-, Cel-CD- and YebF- Nb ${ }^{\text {Stx2 }}$ expression plasmids. Synthetic DNA fragments 52 composed of the following E. coli codon optimized components [attB1-RBS-(OsmY or N20-Cel$53 \mathrm{CD}$ or Cel-CD or Yeb)-FL-2xNb ${ }^{\mathrm{St} 2}$-attB2] were introduced into pDONR221 via BP reactions

54 followed by pDSW206-ccdB-3xFLAG ${ }^{2}$ via LR reactions to create pDSW206-OsmY-Nb ${ }^{\text {Stx2 }}$, 55 pDSW206-N20-CelCD-Nb ${ }^{\mathrm{St} \times 2}$, pDSW206-CelCD-NbSt×2 and pDSW206-YebF-NbSt×2.

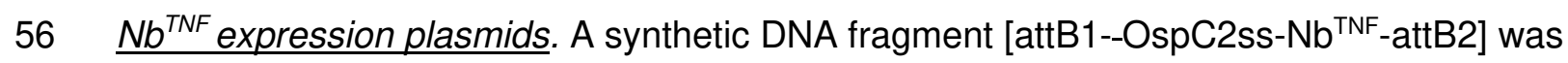

57 introduced into pDONR221 via a BP reaction followed by pDSW206-ccdB-FLAG via an LR 58 reaction to create pDSW206-OspC2ss-Nb $\mathrm{NNF}^{\mathrm{TN}}$. A DNA fragment composed of homodimer $\mathrm{Nb}^{\mathrm{TNF}}$

59 fused to an OspC2ss was generated using SOEing PCR with oligomers (P12/P22 and P21/P18)

60 to generate attB1-OspC2ss-2xNb ${ }^{T N F}$-attB2. The resulting fragment was introduced in

61 pDONR221 followed by pDSW206-ccdB-FLAG via BP and LR reaction to generate pDSW206-

62 OspC2-2xNb ${ }^{\text {TNF }}$. pDSW206-PJ23115-OspC2ss-2xNb ${ }^{\text {TNF }}$ and pDSW206-alr-PJ23115-OspC2ss-

$632 \times \mathrm{Nb}^{\mathrm{TNF}}$ were constructed as previously described for $\mathrm{Nb}^{\mathrm{St} \times 2}$.

$64 \underline{V i r B}$ expression plasmids. Entry clones that contain virB under the control of various promoters

65 were obtained via SOEing PCR using two synthetic DNA fragments and oligomers (P23/P24).

66 One synthetic DNA fragment contained a promoter flanked by an upstream attB site and

67 downstream by $40 \mathrm{bp}$ of homology to $\operatorname{vir} B^{1}$. The second DNA fragment contained the open

68 reading frame of virB codon-optimized for expression in E. coli with an upstream RBS and a

69 downstream stop codon followed by an attB site. The RBS Calculator tool version $1.1^{1}$, with

70 organism option as E. coli str. K-12 substr. MG1655, was used to choose the RBS. The 
71 resulting DNA fragments were introduced into pDONR221 via a BP reaction followed by

72 pCMD136-ccdB-FLAG via an LR reaction. pTKIP-PJ23119-virB was generated by PCR

73 amplifying PJ23119-virB from pCGP-PJ23119-virB-Nb with oligomers (P25/P26) that add a 5'

$74 \mathrm{Kpnl}$ site and a 3' rrnB-homology region. Using pCMD136 as a template, the rrnB terminator

75 was amplified with a 5' virB-homology region and a 3' HindIIl site using oligomers (P27/P28).

76 The two fragments were fused together by SOEing PCR using oligomers (P25/P28). The

77 product was digested with $\mathrm{Kpnl}$ and HindIII and ligated into the polylinker of pTKIP-hph.

78 MxiE-Luciferase expression plasmid. A DNA fragment that contains a MxiE-promoter was PCR

79 amplified from pTSAR1Ud2.4s ${ }^{2}$ using oligomers (P29/P30) that add flanking 5' Xhol and 3' Kpnl

80 restriction sites and an RBS. The digested PCR product was ligated into Xhol/Kpn/ pMM534 to

81 generate pMxiE-lux.

\section{Strain construction}

$83 \mathrm{PROT}_{3} E C T-1$ and $P R O T_{3} E C T-2$. A synthetic $1.3 \mathrm{~kb}$ landing pad insertion site was introduced

84 into the atg/gid loci of EcN and E. coli HS to generate EcN-LPatp/gid and EcHS- LP ${ }^{\text {atp/gid }}$ using the

85 lambda red recombination system and the pTKRED helper plasmid ${ }^{3}$. The landing pad fragment

86 was PCR amplified from pTKIP-tetA with oligos (P31/P32) to introduce homology to the atg/gid

87 locus and integration was confirmed by PCR with oligo pairs (P33/P34 and P35/P36). The

88 pmT3SA plasmid which contains the $20 \mathrm{~kb}$ Mxi-Spa operons flanked by LP and Scel sequences

89 was introduced into EcN-LPatp/gid and EcHS-LPatp/gid via triparental mating: donor

90 (DH10ß/pT3SA), helper HB101 (pRK20734) and recipient (EcN- or EcHS-LPatp/gid/pKD46).

91 pKD46-cured EcN- and HS-LPatp/gid containing pT3SA were transformed with pTKRED and

92 landing pad recombination system was used to generate PROT 3 ECT-1 and PROT 3 ECT-2. KAN

93 resistant/TET susceptible transformants were screened for proper integration junctions by PCR

94 with oligo pairs (P33/P37 and P35/P38). 
$\underline{P R O T_{3} E C T-3}$. PROT ${ }_{3} E C T-1$ was modified with a landing pad at its yieN/trkB locus to create

$96 \mathrm{PROT}_{3} \mathrm{ECT}-1$-LPyie/trk. By PCR, the landing pad with appropriate homology regions was

97 amplified with oligos (P39/P40) and its integration was confirmed with P41/P34 and P42/P36.

$98 \mathrm{PROT}_{3}$ ECT-1-LPyietrk was transformed with pTKred and pTKIP-PJ23119-virB and the landing

99 pad platform was used to introduce the VirB expression construct into the chromosome.

100 Integration was confirmed by PCR with oligos P43/P28 and P44/P27.

$101 \underline{P_{R O T} E C T-4}$. After first resolving the $\mathrm{KAN}^{\mathrm{R}}$ marker previously used to introduce the Mxi-Spa

102 operons into $\mathrm{PROT}_{3} \mathrm{ECT}-1$ using the FLP recombinase, the lambda red recombination system

103 was used to sequentially delete alr and dadX from PROT 3 ECT-3 using oligomers (P45/P46 and

$104 \mathrm{P} 47 / \mathrm{P} 48)^{5}$. The $\mathrm{KAN}^{\mathrm{R}}$ was removed from the alr locus, before proceeding to delete $\mathrm{dad} X$.

105 Deletions were confirmed by PCR with oligomers (P49/P50 and P51/P52, respectively).

\section{Gentamicin protection assay.}

107 HCT8 cells were seeded in 96-well plates $\left(4 \times 10^{4}\right.$ cells per well) for $18 \mathrm{~h}$ prior to exposure to

108 bacteria. Bacteria grown overnight with aeration at $37^{\circ} \mathrm{C}$ were back-diluted and subcultured for

109 one hour before the addition of IPTG (1 mM). One hour later, the HCT8 cells were infected at an

$110 \mathrm{MOI}$ of 100 . After $30 \mathrm{~min}$, gentamicin $(50 \mu \mathrm{g} / \mathrm{mL})$ was added, and 30 minutes later, cells were

111 lysed with $1 \%$ triton X-100 in PBS. Bacteria were plated and enumerated. Percentage of

112 internalized bacteria was determined by calculating the ratio of gentamicin resistant bacteria to

113 the initial inoculum.

\section{Liquid secretion assays.}

115 Liquid Secretion assays were performed as previously described ${ }^{36}$ with some modifications.

116 Overnight cultures of $E$. coli grown in LB (Luria broth) were back diluted 1:50. Once cultures

117 reached $\mathrm{OD}_{600}$ of 1.2-1.5, the bacteria were pelleted and resuspended in fresh media or PBS 
118 and incubated for the times indicated. IPTG (1 mM, Sigma) was added when studying Ptrc

119 regulated virB and/or $n b$. When indicated, bacteria were exposed to $10 \mu \mathrm{M}$ Congo red (Sigma).

120 After designated periods of time, total cell and supernatant fractions were separated by

121 centrifugation at $20,000 \mathrm{~g}$ for $2 \mathrm{~min}$. The cell pellet was taken as the total cell fraction. The

122 supernatant fraction was subjected to a second centrifugation step. To account for differences in

123 bacterial titers, the volume of protein loading dye $(40 \%$ glycerol, $240 \mathrm{mM} \mathrm{Tris} / \mathrm{HCl} \mathrm{pH} 6.8,12 \%$

124 SDS, $0.04 \%$ bromophenol blue, $5 \%$ beta-mercaptoethanol) used to resuspend each sample

125 was normalized by the $\mathrm{OD}_{600}$ reading of the slowest growing culture. For some assays, as

126 indicated in the text, samples were not normalized. The pellet was resuspended in $100 \mu \mathrm{L}$ or

127 more protein loading dye, depending on the $\mathrm{OD}_{600}$, and $5 \mu \mathrm{L}$ was loaded onto a $10 \%$ SDS-

128 PAGE gel for analysis. Proteins in the supernatant were precipitated with trichloroacetic acid

$129(\mathrm{TCA})(10 \% \mathrm{v} / \mathrm{v})$ and resuspended in $50 \mu \mathrm{L}$ or more protein loading dye, depending on the $130 \mathrm{OD}_{600}$. Ten microliters of TCA-precipitated supernatant samples were loaded onto a $12 \%$ SDS-

131 PAGE gel for analysis. Proteins were transferred to nitrocellulose membranes and

132 immunoblotted with mouse anti-FLAG (1:10,000, clone M2, Sigma,), mouse anti-HA (1:1000, 133 clone 16B12, Biolegend) or rabbit anti-GroEL (1:100,000, G6532, Sigma). Alternatively, SDS-

134 PAGE gels were stained with GelCode ${ }^{\mathrm{TM}}$ Blue Stain Reagent (Thermo Fisher Scientific), per the 135 manufacturer's instructions.

136 Solid plate secretion assay.

137 Solid plate secretion assays were performed as previously described. Briefly, single colonies 138 grown overnight in 96 well plates were quad spotted onto a solid agar plate ${ }^{7}$. After overnight 139 growth, a robotic 384-pin tool is used to transfer equivalent amounts of bacteria to a second 140 media containing plate over which a nitrocellulose membrane was immediately laid. After 6 hrs 141 at $37^{\circ} \mathrm{C}$, the membrane was removed, washed, and immunoblotted for protein of interest.

142 Fecal shedding assay. 
143 Fecal pellets were collected and weighed. A 10x volume of PBS was added and the samples

144 homogenized by pipetting and mashing using wide mouth pipette tips before being serially

145 diluted and plated on MacConkey agar plates with antibiotics. After overnight incubation at 37

$146{ }^{\circ} \mathrm{C}$ colonies were counted and the total number of CFU estimated.

147 In vitro luciferase monitoring.

148 In a 96 well white plate, 1:100 dilutions of overnight bacterial cultures were incubated at $37^{\circ} \mathrm{C}$

149 with shaking for $5 \mathrm{~h}$. Readings were performed on a SpectraMax i3x Multi-Mode Microplate

150 Detection Platform (Molecular devices).

151 In vivo luminescence assays.

152 To image luciferase-expressing bacteria in the GI tract, mice pre-treated for 1 day with

153 kanamycin $(1 \mathrm{~g} / \mathrm{L})$ and spectinomycin $(2 \mathrm{~g} / \mathrm{L})$ in their drinking water were orally gavaged with $10^{8}$

154 CFU of PROT3EcT-3 or EcN harboring the constitutive luciferase or MxiE reporter plasmids.

155 After sacrificing the mice, the cecum, colon and small intestine were harvested, the contents

156 gently removed, and the tissues placed on a black mat for imaging using an IVIS Spectrum CT.

157 Tissues were imaged using a luminescence filter, with field of view (FOV) $=D(22.2 \mathrm{~cm})$, fstop =

1581 and large binning. Data was analyzed using Living Image Software 4.3.1.

159 Discovery and characterization of Nbs targeting murine TNFa.

160 Two alpacas were each immunized once with $200 \mu \mathrm{g}$ murine (m)TNF- $\alpha$ (Biolegend 575204) in

$161 \mathrm{CpG}$ /alum adjuvant, followed by four boosters with $100 \mu \mathrm{g}$ mTNFa in alum adjuvant only. Nb

162 display phage library construction, panning and screening were done as previously described ${ }^{8}$.

163 Given that the number of unique Nb families obtained in first panning was low, it was repeated

164 with mTNFa bound to JTT-B10 Nb, a Nb obtained in the initial screen. This second panning

165 yielded 20 unique mTNFa-binding Nb families. The coding sequences of representative 
members of each anti-TNF-a family were introduced into (Novagen) and expressed as

167 thioredoxin, 6-His, E-tag fusion proteins in E. coli Rosetta-gami 2 (DE3) pLacl (Novagen) as

168 fusions to thioredoxin to promote localization to periplasm and to hexahistadine (His6) to

169 facilitate purification using standard Ni-IMAC chromatography methods, and a carboxyl terminal

170 E-tag for detection. Based on ELISA ${ }^{8}, 10$ unique Nbs with $10 \mathrm{nM}$ or better apparent affinities

171 were selected for further analyses (Table S1) (Fig. S6A).

172 A competition study was conducted to determine whether any of the other unique $\mathrm{Nb}$

173 bound to epitopes not recognized by JTT-B10 by performing replicate dilution ELISAs in which

174 the only variation was that one set of ELISAs contained $20 \mu \mathrm{g} / \mathrm{ml}$ of the JTT-B10 $\mathrm{Nb}$ protein as a

175 competitor in which the E-tag detection tag was replaced with a myc tag (Figure S6c). The study

176 identified three $\mathrm{Nb}$ families that bind to epitopes not competed by JTT-B10 (Table S1).

\section{L929 cell cytotoxicity assay}

$\mathrm{Nbs}^{\mathrm{TNF}}$ and bacterial supernatants were assessed for their ability to neutralize mTNFa

180 using a TNFa-induced cytotoxicity assay in L929 cells, as previously described ${ }^{9}$. Briefly, 100

$181 \mu \mathrm{l} /$ well of murine fibroblast L929 cells seeded in 96 -well plates $\left(5 \times 10^{4}\right.$ cells/well). After

182 overnight incubation, the culture medium was replaced with serial dilutions of bacterial

183 supernatants or purified Nb prepared in RPMI media containing a final concentration of 1.0

$184 \mu \mathrm{g} / \mathrm{ml}$ actinomycin D and $4 \mathrm{ng} / \mathrm{mL}$ murine TNF- $\alpha$ (Biolegend 575204). Plates were then

185 incubated at $37^{\circ} \mathrm{C}$ for $24 \mathrm{~h}$ after which an MTT assay was performed as per the manufacturer's

186 instructions (Trevigen 4890-25-K). The only mTNFa neutralizing Nb was JTT-B10. (Table S1).

187 TNBS mouse model of colitis and treatment protocol.

188 Time points and doses for all treatments and administrations are indicated in the Figures and

189 text. TNBS (Sigma, 92822) was diluted to $20 \mathrm{mg} / \mathrm{mL}$ in ethanol $(50 \% \mathrm{v} / \mathrm{v})$ and $100 \mu \mathrm{l}$

190 administered via enema by inserting a 3.5 French catheter (Utah Medical Products) $3 \mathrm{~cm}$ into 
191 the colon. Bacterial strains were prepared as described and administered via oral gavage or

192 enema. Anti-TNF mAb (BioxCell, clone TN3-19.12) was administered intraperitoneally (i.p.).

193 Mice were euthanized by $\mathrm{CO}_{2}$ overdose. Upon sacrifice, blood was harvested by cardiac bleed,

194 the Gl tracts excised, and colon lengths measured. Blood was collected into serum separator

195 tubes, spun for $5 \mathrm{~min}$ at $5000 \mathrm{rpm}$ and serum stored at $-20^{\circ} \mathrm{C}$. The colon was cut longitudinally,

196 the contents removed and the tissue dissected. Half of the tissue was fixed using $4 \%$

197 paraformaldehyde (PFA) overnight at $4^{\circ} \mathrm{C}$ for histology. The other half was homogenized in 1

$198 \mathrm{~mL}$ of PBS containing 1x HALT protease inhibitor cocktail (Thermo Scientific) before being

199 centrifuged for $10 \mathrm{~min}$ at $20,000 \mathrm{~g}$ and the supernatant stored at $-20^{\circ} \mathrm{C}$ for later analysis by

200 ELISA.

201 Cytokine and Nb ELISAs.

202 The concentrations of mouse TNFa, IL-10, IL-6 (BioLegend) were quantified by ELISA per the 203 manufacturer's instructions. The anti-Nb ELISA was performed as previously described ${ }^{9}$.

\section{Histology.}

205 PFA-fixed colon tissue was transferred to $70 \%$ ethanol before processing by routine paraffin 206 embedding, sectioning and H\&E staining by the DF/HCC Rodent Histopathology Core. A 207 pathologist (J.N.G.), blinded to experimental parameters, determined colitis scores. Each of the 208 following four histologic parameters were scored as absent (0), mild (1), moderate (2), or severe 209 (3): mononuclear cell infiltration, polymorphonuclear cell infiltration, epithelial hyperplasia, and 210 epithelial injury. The scores for the parameters were summed to generate the cumulative 211 histologic colitis score ${ }^{10}$. The cumulative histologic colitis score was then multiplied by an extent 212 score, indicating the proportion (\%) of colon involved by colitis: (1) < 10\%; (2) 10\%-25\%; (3) $21325 \%-50 \%$; (4) > 50\%. Images were captured at $10 \times$ or $40 \times$ magnification with a Nikon Eclipse 214 NI-U and NSI-Element Basic Research software (Nikon). 
216 Statistical analyses were performed using GraphPad Prism v.8.3.0. Data are shown as mean \pm

217 SEM as noted. Data were analyzed using a Kruskal-Wallis test with Dunn's multiple correction

218 test or a two-way ANOVA with Tukey's test. A p-value $<0.05$ was considered statistically

219 significant.

\section{References}

222

1. Schmitz, A.M., Morrison, M.F., Agunwamba, A.O., Nibert, M.L. \& Lesser, C.F. Protein interaction platforms: visualization of interacting proteins in yeast. Nature Methods 6 , 500-502 (2009).

2. Mou, X., Souter, S., Du, J., Reeves, A.Z. \& Lesser, C.F. Synthetic bottom-up approach reveals the complex interplay of Shigella effectors in regulation of epithelial cell death. Proceedings of the National Academy of Sciences of the United States of America 115, 6452-6457 (2018).

3. Kuhlman, T.E. \& Cox, E.C. Site-specific chromosomal integration of large synthetic constructs. Nucleic Acids Res 38, e92-e92 (2010).

4. Leong, S.A., Ditta, G.S. \& Helinski, D.R. Heme biosynthesis in Rhizobium. Identification of a cloned gene coding for delta-aminolevulinic acid synthetase from Rhizobium meliloti. Journal of Biological Chemistry 257, 8724-8730 (1982).

5. Datsenko, K.A. \& Wanner, B.L. One-step inactivation of chromosomal genes in Escherichia coli K-12 using PCR products. Proceedings of the National Academy of Sciences of the United States of America 97, 6640-6645 (2000).

6. Reeves, A.Z. et al. Engineering Escherichia coli into a protein delivery system for mammalian cells. ACS Synth Biol 4, 644-654 (2015).

7. Ernst, N.H., Reeves, A.Z., Ramseyer, J.E. \& Lesser, C.F. High-Throughput Screening of Type III Secretion Determinants Reveals a Major Chaperone-Independent Pathway. mBio 9 (2018).

8. Jaskiewicz, J.J., Tremblay, J.M., Tzipori, S. \& Shoemaker, C.B. Identification and characterization of a new $34 \mathrm{kDa}$ MORN motif-containing sporozoite surface-exposed protein, Cp-P34, unique to Cryptosporidium. Int J Parasitol 51, 761-775 (2021).

9. Baarsch, M.J., Wannemuehler, M.J., Molitor, T.W. \& Murtaugh, M.P. Detection of tumor necrosis factor alpha from porcine alveolar macrophages using an L929 fibroblast bioassay. J Immunol Methods 140, 15-22 (1991).

10. Garrett, W.S. et al. Communicable ulcerative colitis induced by T-bet deficiency in the innate immune system. Cell 131, 33-45 (2007). 


\section{Supplementary Figure Legends}

256

257

258

259

260

261

262

263

264

265

266

267

268

269

270

271

272

273

274

275

276

277

278

279

280

281

282

283

284

285

286

287

288

289

290

Figure S1. Schematic overview and evidence that PROT $\mathrm{PCCT}_{3}-2$ assembles a functional T3SA. The Ipa-Mxi-Spa and Mxi-Spa operons (a) were captured and integrated into the chromosome of E. coli resulting in mT3_Ipa-Mxi-Spa and mT3_Mxi-Spa, respectively (b). The absence of the Ipa operons enables mT3_Mxi-Spa to secrete proteins into its surroundings as opposed to injecting them into host cells. (c) Secretion of OspC2-FLAG by the indicated strains was monitored by a $6 \mathrm{hr}$ liquid secretion assay. Immunoblots of FLAG-tagged OspC2 and GroEL. Data in each panel is representative of results from at least 2 independent experiments. $\mathrm{P}=$ whole cell pellet lysates, $\mathrm{S}=$ supernatant fractions.

Figure S2. Comparison of secretion of $\mathrm{Nb}_{\text {by }} \mathrm{PROT}_{3} \mathrm{EcT}$ and native E. coli carrier proteins. (a) $6 \mathrm{~h}$ liquid secretion assays monitoring the secretion of FLAG-tagged NbStx2 dimers fused to designated native $\mathrm{E}$. coli carrier protein sequences in EcN and BL21 E. coli (b). $\mathrm{FL}=$ full length Cel-CD. Data in each panel is representative of results from at least 2 independent experiments.

Figure S3. PROT 3 EcT-4 stably maintains its alr-plasmid (a) Plasmid retention rate in strains indicated. Bacterial cultures were back diluted daily for 7 days and grown in LB media without antibiotics. Each day cultures were sampled and plated on LB media to quantify total bacteria and LB/ampicillin plates to quantify bacteria that had retained their plasmid. Data in each panel is representative of results from at least 2 independent experiments. CFU = colony forming units.

Figure S4. Plate assay of shed bacteria from mice colonized with PROT3EcT-4 and weights of mice treated as indicated. (a-c) $6 \mathrm{~h}$ plate secretion of colonies of PROT3EcT-4 shed from mice at the times indicated. Membranes were removed and probed with an antiFLAG Ab to monitor Nb secretion. (d) Body weight change (\%) of mice inoculated with the strains indicated. Data in each panel is representative of results from at least 2 independent experiments. (b) Data were analyzed using two-way ANOVA with Tukey's post-hoc test. ns = not significant.

Figure S5. In vitro validation of pMM534 and pMxiE-Lux reporters, and levels of bacteria in mice inoculated with PROT $_{3}$ EcT-3 and treated with antibiotics. (a,f) Luminescence readings of the strains indicated. Bacteria were grown for $18 \mathrm{~h}$, back diluted 1:100 into plates containing media. At $2 \mathrm{~h}$ post back dilution luminescence and $\mathrm{OD}_{600}$ were recorded. (b,e) IVIS images of the indicated strains that have been spread on agar plates or grown in liquid culture for $18 \mathrm{~h}$. (d) Schematic of pMxiE-Lux reporter. VirB promotes the expression of MxiE which is activated when bound to IpgC. For these assays, a plasmid that encodes IpgC was introduced into the strains, as is encoded in the Ipa operon, which is absent from PROT $_{3} E C T$. RLU = relative luminescence units. $\mathrm{OD}=$ optical density. 
Figure S6. $\mathbf{N b}^{\mathrm{TNF}}$ discovery and in vitro testing. (a) $\mathrm{Nb}^{\mathrm{TNF}}$ DNA sequences. (b) Affinities of purified $\mathrm{Nb}^{\mathrm{TNF}}$ were measured by ELISA. (c) Functional activity of the $\mathrm{Nb}^{\mathrm{TNF}}$ was measured using the TNF-L929 killing assay.

Figure S7. TNBS colitis is TNFa dependent. (a) Study design. BALB/c mice were treated with TNBS as before and administered anti-TNFa monoclonal antibody (mAb) intraperitoneally (i.p.) at the times indicated and sacrificed at 5 days post TNBS. (b) Body weight change (\%). (c) Colon length. (d) Histologic colitis scores. (e-g) TNFa levels were measured in the indicated samples by ELISA. Data are representative of at least 2 experiments with $n=3-5$ mice per group and are presented as individual values and mean \pm SEM (b) or mean \pm SEM (c-g). Data were analyzed using two-way ANOVA with Tukey's post hoc test (b) or a Kruskal-Wallis test with Dunn's multiple correction test (c-g). ${ }^{*}, \mathrm{P}<0.05 ;{ }^{* *}, \mathrm{P}<0.01$; $^{* * *}, \mathrm{P}<0.001$ denotes comparison to PBS group or as indicated.

Figure S8. TNF-PROT ${ }_{3}$ EcT and $-\mathrm{T}_{3}$ EcT-3 are efficacious in the TNBS model when administered via enema. (a) Study design. BALB/c mice were treated with TNBS as before and administered PBS or an inoculum of $10^{8} \mathrm{CFU}$ of $\mathrm{T}_{3} \mathrm{EcT}_{-3}$, TNF-T $\mathrm{T}_{3} \mathrm{EcT}-3, \mathrm{PROT}_{3} \mathrm{EcT}-4$ or TNF-PROT ${ }_{3}$ EcT- 4 via enema administration at the times indicated and were sacrificed at 5 days post TNBS. (b) Shed bacteria. (c) Body weight change (\%). (d) Colon length. (e) Histologic colitis scores. (f) $6 \mathrm{~h}$ liquid secretion assays monitoring the secretion of FLAG-tagged $\mathrm{Nb}^{\mathrm{TNF}}$ and $\mathrm{Nb}^{\text {Stx2 }}$ by the indicated strains. (g) Plate secretion assay of shed bacteria. Data were combined from 2 independent from at least $n=2$ experiments with 3-5 mice per group and are presented as individual values and mean \pm SEM (d-e) or mean \pm SEM (b-c). Data were analyzed using two-way ANOVA with Tukey's post hoc test $(b, c)$ or a Kruskal-Wallis test with Dunn's multiple correction test (d-e). *, $\mathrm{P}<0.05 ;{ }^{* *}, \mathrm{P}<0.01 ;{ }^{* *}, \mathrm{P}<0.001$ denotes comparison to PBS group or as indicated;, $\mathrm{P}<0.05^{\# \#, ~} \mathrm{P}<0.01$; \#\#, $\mathrm{P}<0.001$ denotes comparison to $\mathrm{PROT}_{3} \mathrm{EcT}-4$. of treated mice. $\mathrm{Nb}^{\text {TNF }}$ levels were measured by ELISA in (a) serum, (b) colon tissue homogenates and (c) colon contents of mice receiving the strains or treatments indicated. Dotted line indicates maximal background in the ELISA. Data were combined from 2 independent experiments. 
Figure S1

a
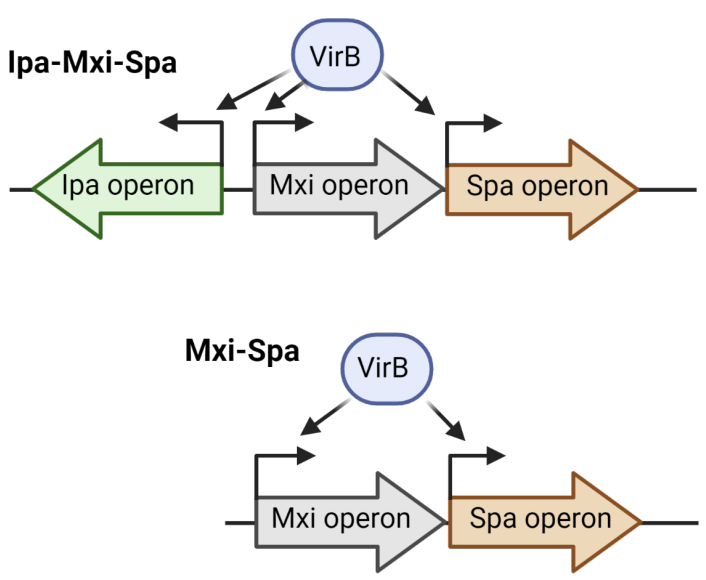

b
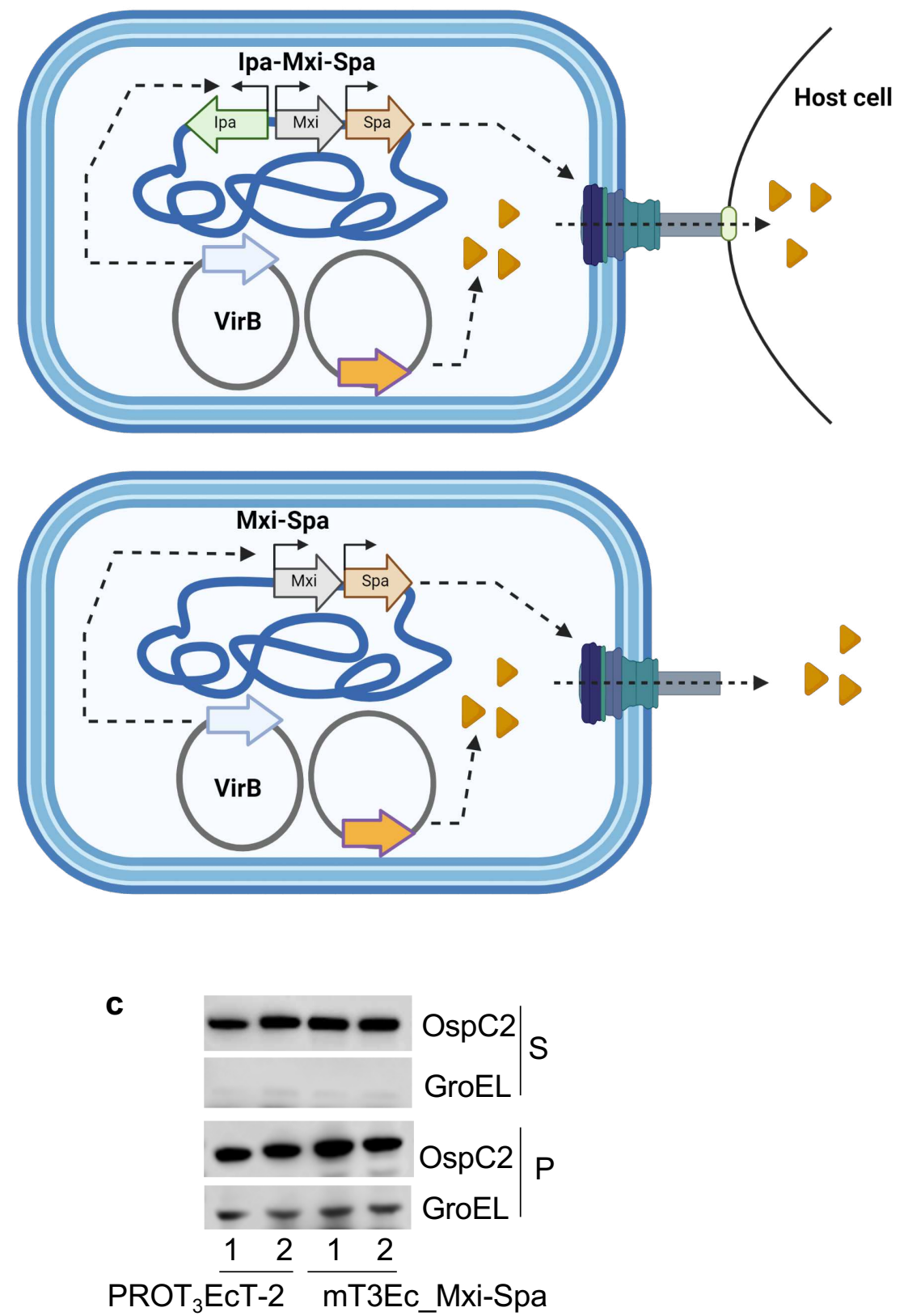
Figure S2

a

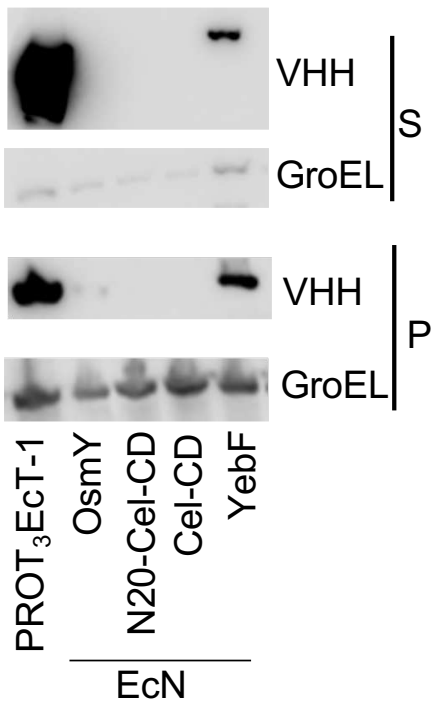

b

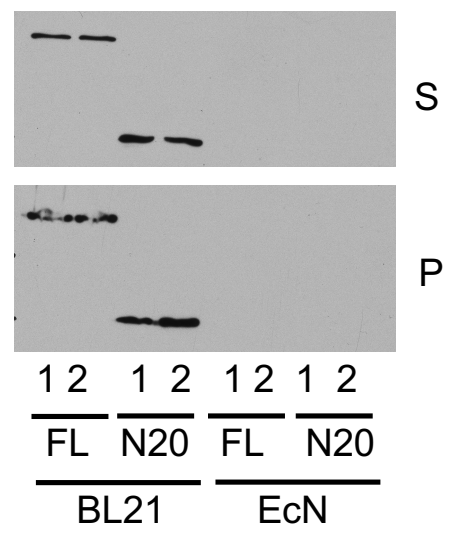


Figure S3

a

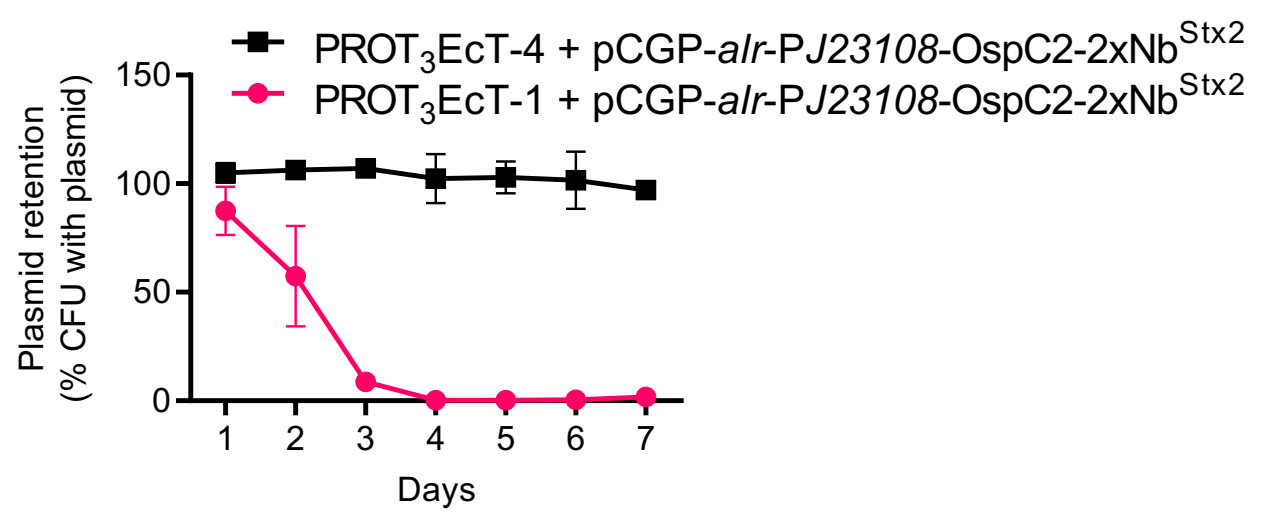


Figure S4

a

Colony

\begin{tabular}{|c|c|c|c|c|c|c|c|c|c|c|c|c|}
\hline $2 d p i$ & 1 & 2 & 3 & 4 & 5 & 6 & 7 & 8 & 9 & 10 & 1 & 2 \\
\hline \multirow{4}{*}{$\begin{array}{l}\stackrel{D}{\mathscr{N}} \\
\stackrel{\mathscr{S}}{D} \\
\stackrel{0}{\Sigma}\end{array}$} & $\begin{array}{ll}- & 0 \\
- & 0\end{array}$ & 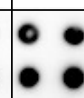 & & •? & 0 & & • & ? & 0 & • & $\begin{array}{|ll|}\bullet & \bullet \\
\bullet & \bullet \\
\end{array}$ & $\bullet \bullet$ \\
\hline & $\begin{array}{ll}1 & 0 \\
0 & 0\end{array}$ & $\begin{array}{ll}0 & 0 \\
0 & \bullet\end{array}$ & $\bullet \bullet$ & $\begin{array}{ll}0 & \bullet \\
\bullet & 0\end{array}$ & $\begin{array}{ll}0 & 0 \\
0 & 0\end{array}$ & $\bullet$ & $\bullet$ & $\begin{array}{ll}0 & 0 \\
\bullet & \bullet\end{array}$ & $\begin{array}{ll}0 & 0 \\
\bullet & 0\end{array}$ & - & \multicolumn{2}{|c|}{$\begin{array}{l}\text { Inoculum } \\
\text { strain }\end{array}$} \\
\hline & $\begin{array}{ll}0 & 0 \\
0 & 0\end{array}$ & $\mid \begin{array}{cc}0 & \bullet \\
0 & \bullet\end{array}$ & & & $\bullet$ & $\bullet$ & $\bullet$ & $\begin{array}{ll}\bullet & \bullet \\
\bullet & 0\end{array}$ & $\begin{array}{ll}\bullet & \bullet \\
\bullet & \bullet\end{array}$ & • & & \\
\hline & 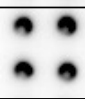 & $\mid \begin{array}{ll}0 & \bullet \\
0 & 0\end{array}$ & $\bullet$ & & $\bullet \bullet$ & $\begin{array}{ll}\bullet & \bullet \\
\bullet & \bullet\end{array}$ & $\bullet$ & $\bullet \bullet$ & $\bullet \bullet$ & $\bullet$ & & \\
\hline
\end{tabular}

Colony

b

\begin{tabular}{|c|c|c|c|c|c|c|c|c|c|c|}
\hline $5 d p i$ & 1 & 2 & 3 & 4 & 5 & 6 & 7 & 8 & 9 & 10 \\
\hline 1 & $\bullet \bullet$ & $\bullet$ & $\bullet$ & $\begin{array}{l}\bullet \bullet \\
\bullet \bullet\end{array}$ & $\begin{array}{ll}0 & 0 \\
0 & 0\end{array}$ & & & $\bullet$ & $\begin{array}{ll}\bullet & \bullet \\
0 & 0\end{array}$ & $\begin{array}{ll}\bullet & \bullet \\
\bullet & \bullet\end{array}$ \\
\hline$\Phi$ & $\bullet \bullet$ & $\bullet \bullet$ & $\bullet$ & $\because 0$ & $\because$ & $\therefore c$ & & $\bullet$ & $\bullet \bullet$ & $60^{20^{* k}}$ \\
\hline 3 & $\bullet$ & $\bullet \bullet$ & 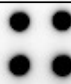 & & & $\bullet \bullet$ & $\bullet$ & $\begin{array}{ll}\bullet \bullet \\
\bullet\end{array}$ & $\begin{array}{ll}\bullet & \bullet \\
\bullet & \bullet\end{array}$ & $\bullet \bullet$ \\
\hline 4 & & 0 & $\bullet$ & & $\bullet \bullet$ & $\bullet$ & 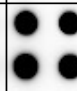 & $\bullet$ & $\begin{array}{ll}\bullet & \bullet \\
\bullet & \bullet\end{array}$ & $\bullet \bullet$ \\
\hline
\end{tabular}

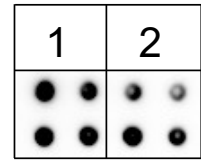

Inoculum

strain
Colony

c

\begin{tabular}{|c|c|c|c|c|c|c|c|c|c|c|c|c|}
\hline $7 d p i$ & 1 & 2 & 3 & 4 & & 5 & 6 & 7 & & 8 & 9 & 10 \\
\hline \multirow{4}{*}{$\begin{array}{l}0 \\
\stackrel{0}{0} \\
\stackrel{0}{\Sigma}\end{array}$} & & & & & c & $\odot$ & - & o & & $\begin{array}{ll}0 & 0 \\
0 & 0\end{array}$ & & \\
\hline & $\begin{array}{ll}0 & 0 \\
0 & 0\end{array}$ & $\begin{array}{ll}0 & 0 \\
0 & 0\end{array}$ & • & & c & c & $\begin{array}{ll}0 & 0 \\
0 & 0\end{array}$ & & & $\begin{array}{ll}0 & 0 \\
0 & 0\end{array}$ & 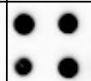 & $\bullet$ \\
\hline & & $\mid \begin{array}{lll}0 & 0 \\
0 & 0\end{array}$ & & $\begin{array}{ll}0 & \bullet \\
0 & \bullet\end{array}$ & c & c & $\bullet$ & & & $\bullet$ & & \\
\hline & $\begin{array}{ll}0 & 0 \\
0 & 0\end{array}$ & $\mid \begin{array}{ll}0 & 0 \\
0 & c\end{array}$ & $\begin{array}{ll}0 & 0 \\
0 & 0\end{array}$ & $\begin{array}{ll}0 & \bullet \\
0 & 0\end{array}$ & 0 & $\bullet$ & $\bullet$ & o & $\bullet$ & $\bullet$ & $\bullet \bullet$ & $\bullet$ \\
\hline
\end{tabular}

d -o- PBS

- EcN

○ $10 \multimap$ PROT $_{3}$ EcT-4l

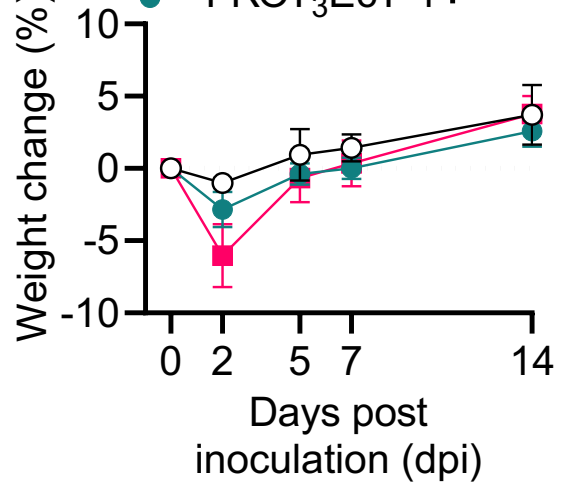

inoculation (dpi)

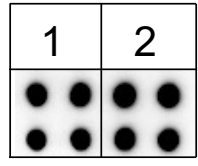

Inoculum

strain 


\section{Figure S5}

a

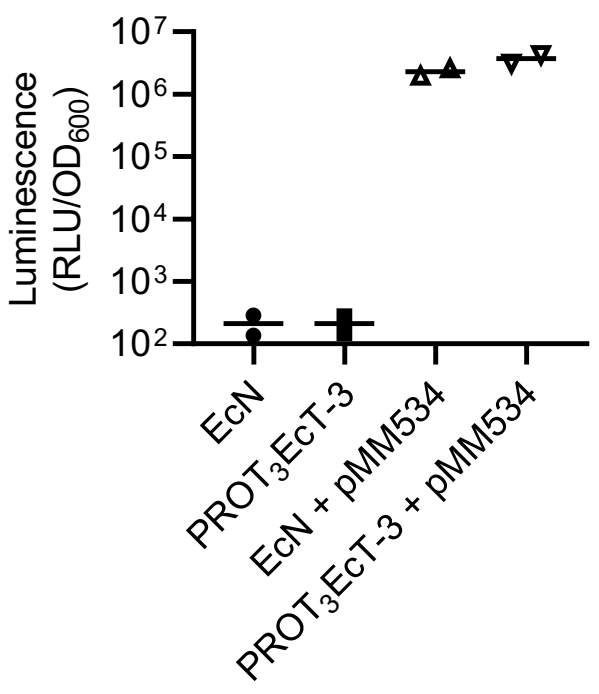

C

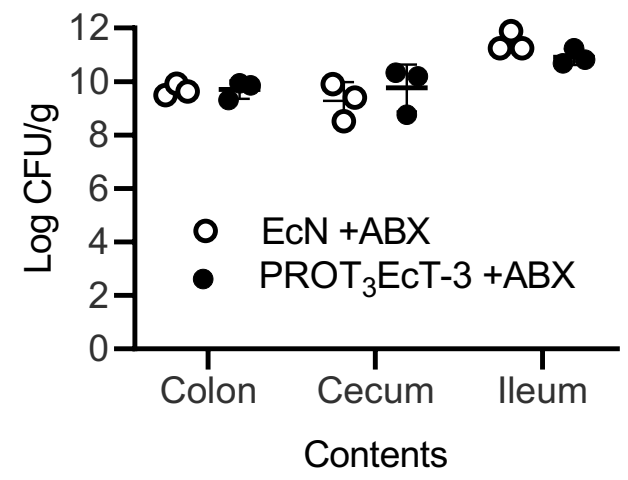

d

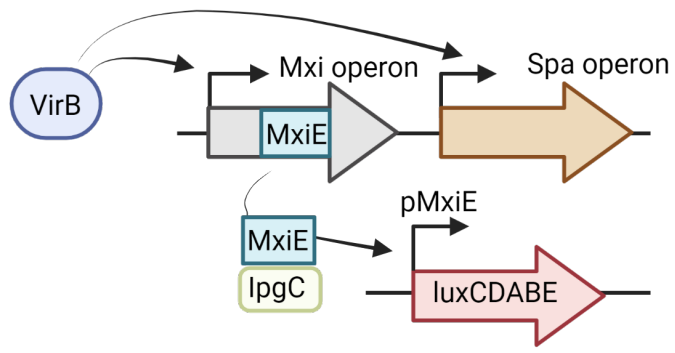

b

(1) $\mathrm{PROT}_{3} \mathrm{EcT}-3$

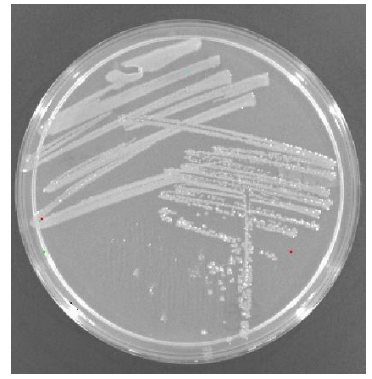

(2) $\mathrm{PROT}_{3} \mathrm{EcT}-3$ pMM534

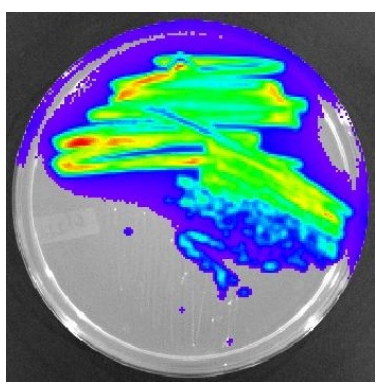

(1)

(2)

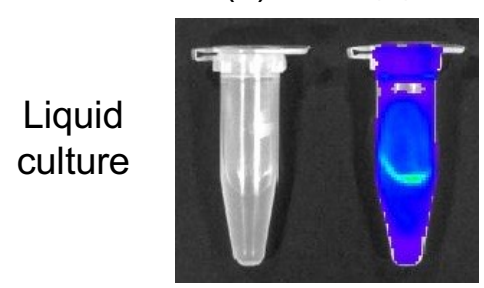

e

$\mathrm{PROT}_{3}$ EcT-3 pMxiE-lux +pNG162-IpgC
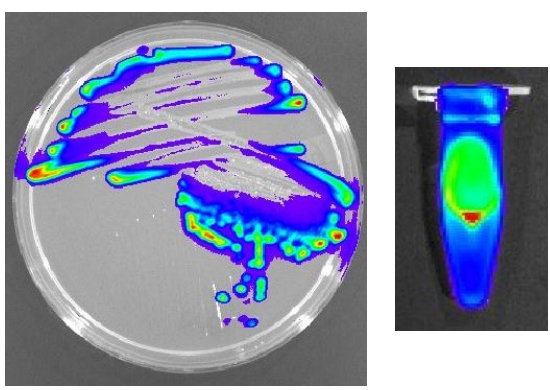

f

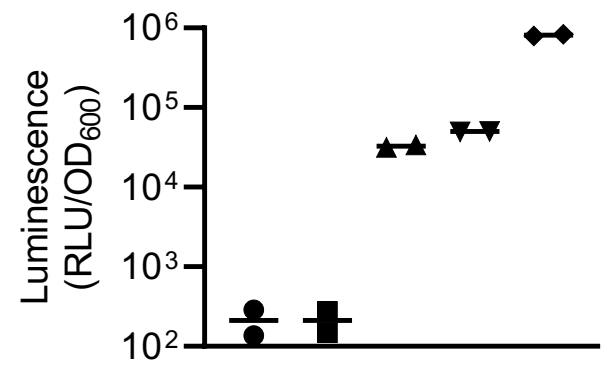

- $\mathrm{EcN}$

- PROT $_{3}$ EcT-3

- $\mathrm{PROT}_{3} \mathrm{EcT}-3+$ pMxiE-Lux

v $\mathrm{EcN}+$ pNG162-IpgC + pMxiE-Lux

- $\mathrm{PROT}_{3} \mathrm{EcT}-3$ + pNG162-IpgC + pMxiE-Lux 


\section{Figure S6}

a $\mathrm{Nb}^{\mathrm{TNF}}$ JTT-B10 JUM-E5 JUM-G5 JUM-G10 JUM-G10 JUN-A1 JUN-B9

JUN-C1

JUN-F5

JUN-G4

JUN-G1 JUN-H5

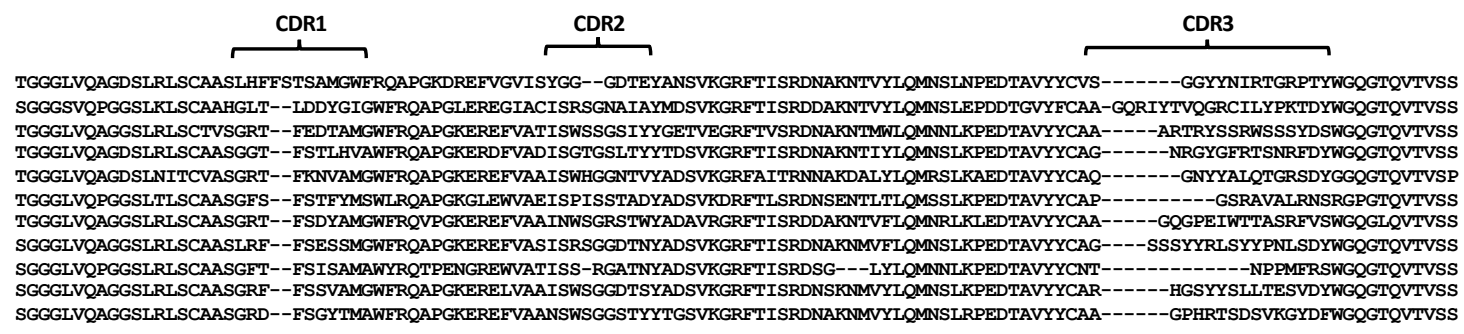

b
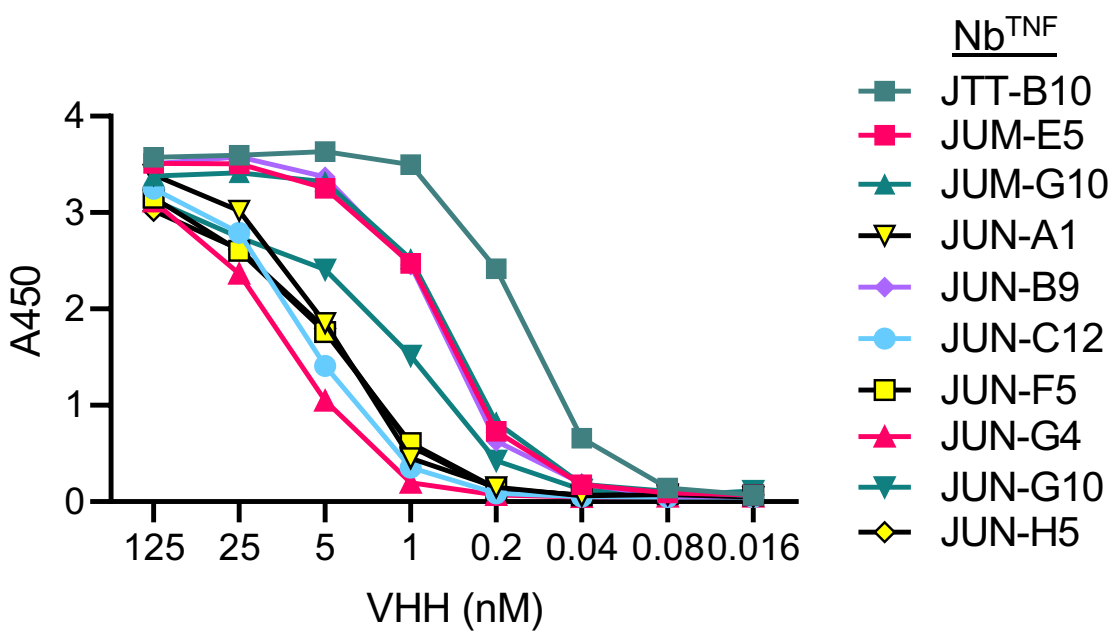

C

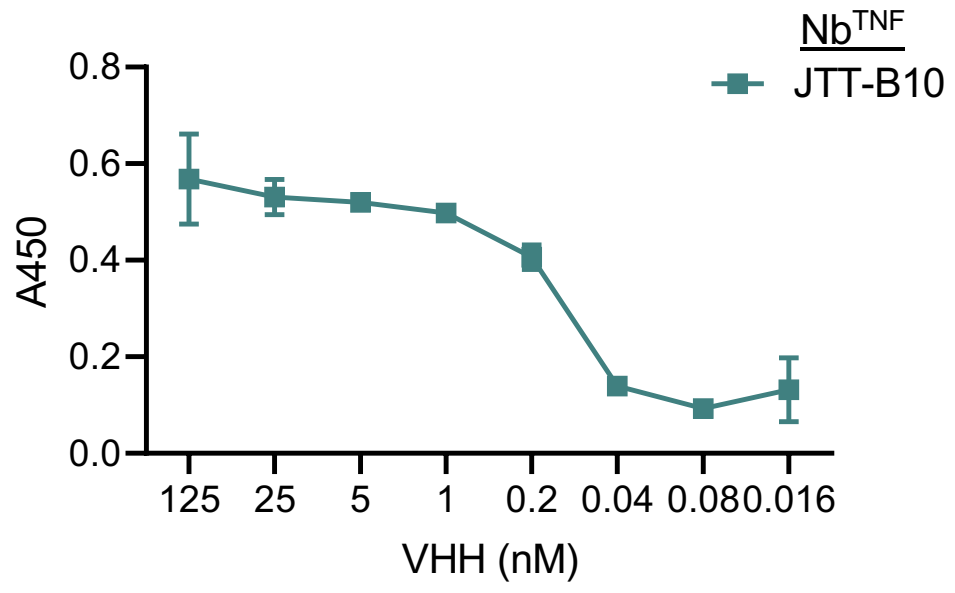


Figure S7

a

aTNF mAb (i.p., $15 \mathrm{mg} / \mathrm{kg}$ )

ØTNBS (enema, 2 mg; 50\% EtOH)

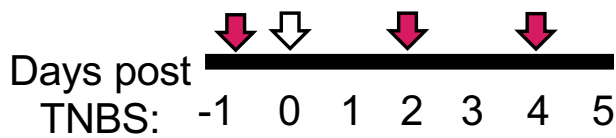

b

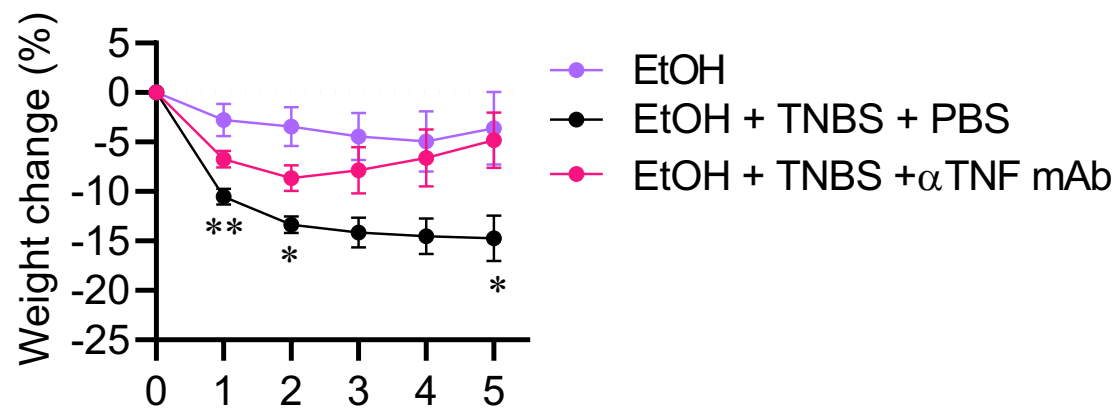

C

d
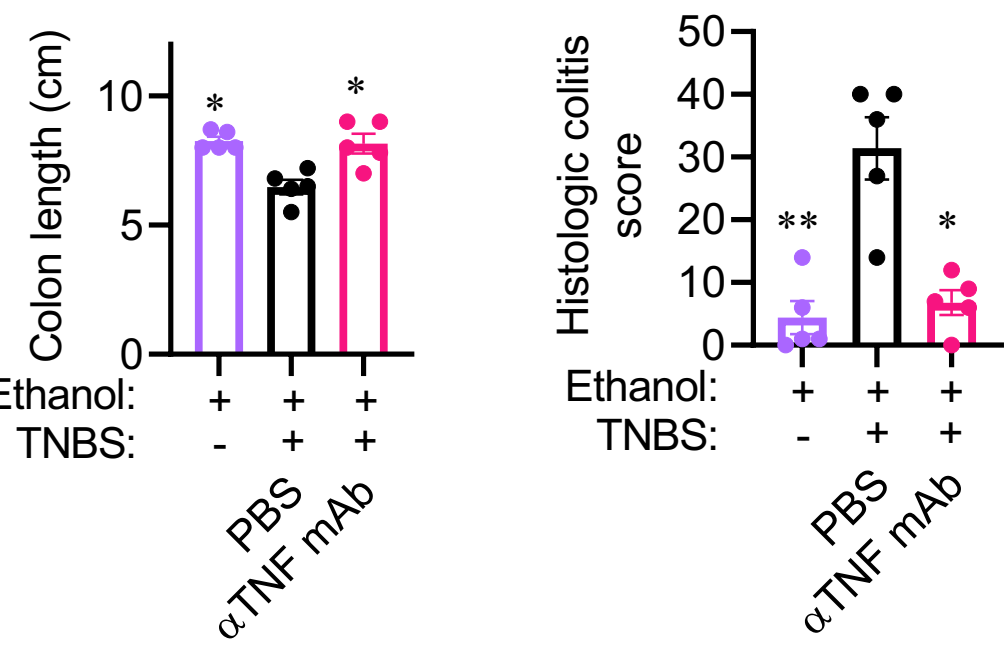

e

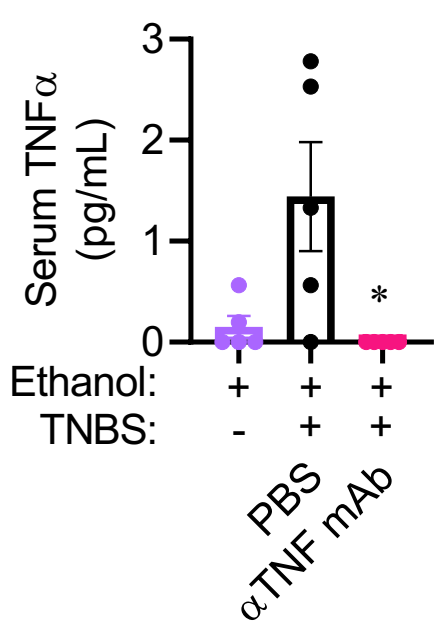

f

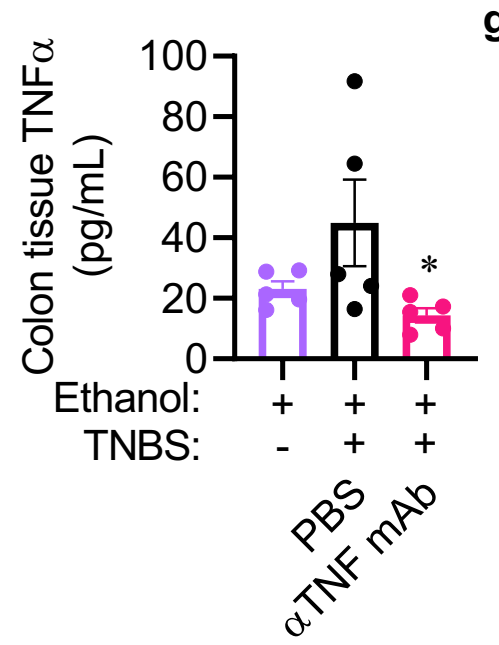

g

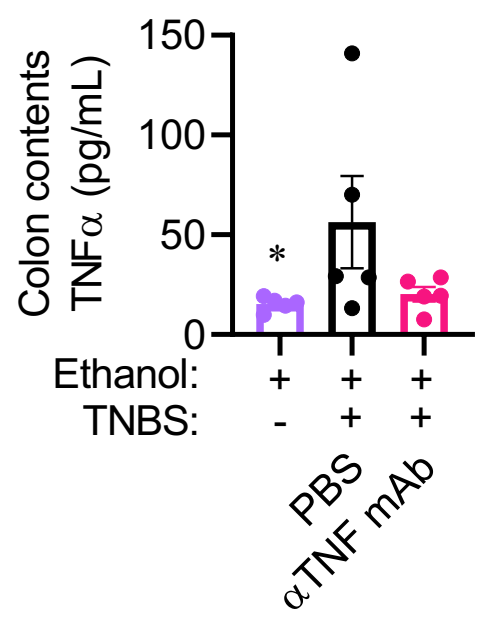


a

$\sqrt{7}$ Strains (enema, $10^{8} \mathrm{CFU}$ )

\TNBS (enema, 2 mg; 50\% EtOH)

Days post

TNBS:

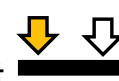

$\begin{array}{lllllll}-1 & 0 & 1 & 2 & 3 & 4 & 5\end{array}$

b

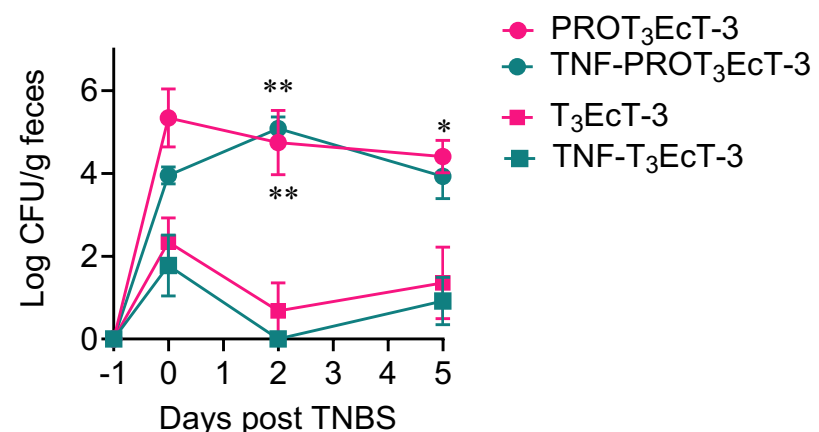

C

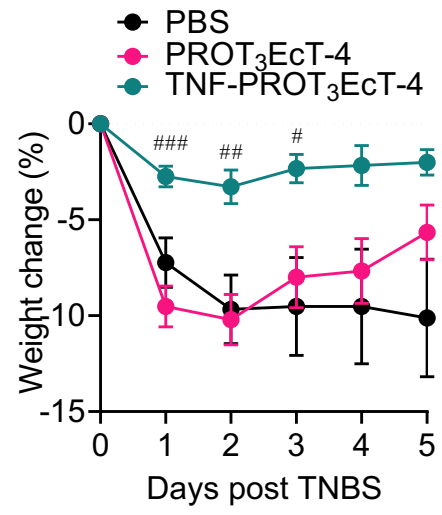

d

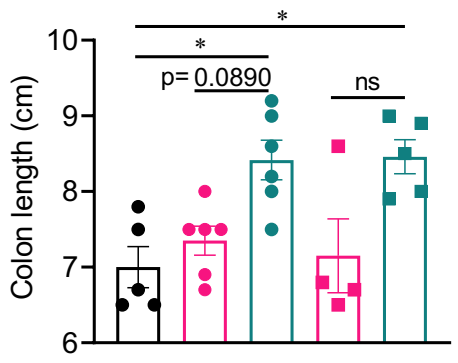

f

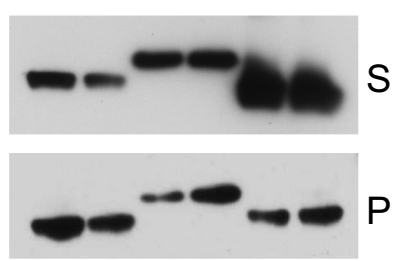

$\frac{1 \quad 2}{{ }_{3} \mathrm{ECT}-4} \frac{12}{\mathrm{Nb}^{\mathrm{ST}} \times 2} \frac{12}{\mathrm{Nb}^{\mathrm{TNF}}}$

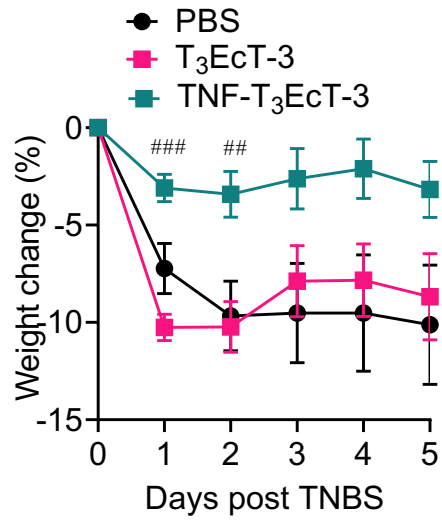

$\mathrm{T}_{3} \mathrm{EcT}-3$ 

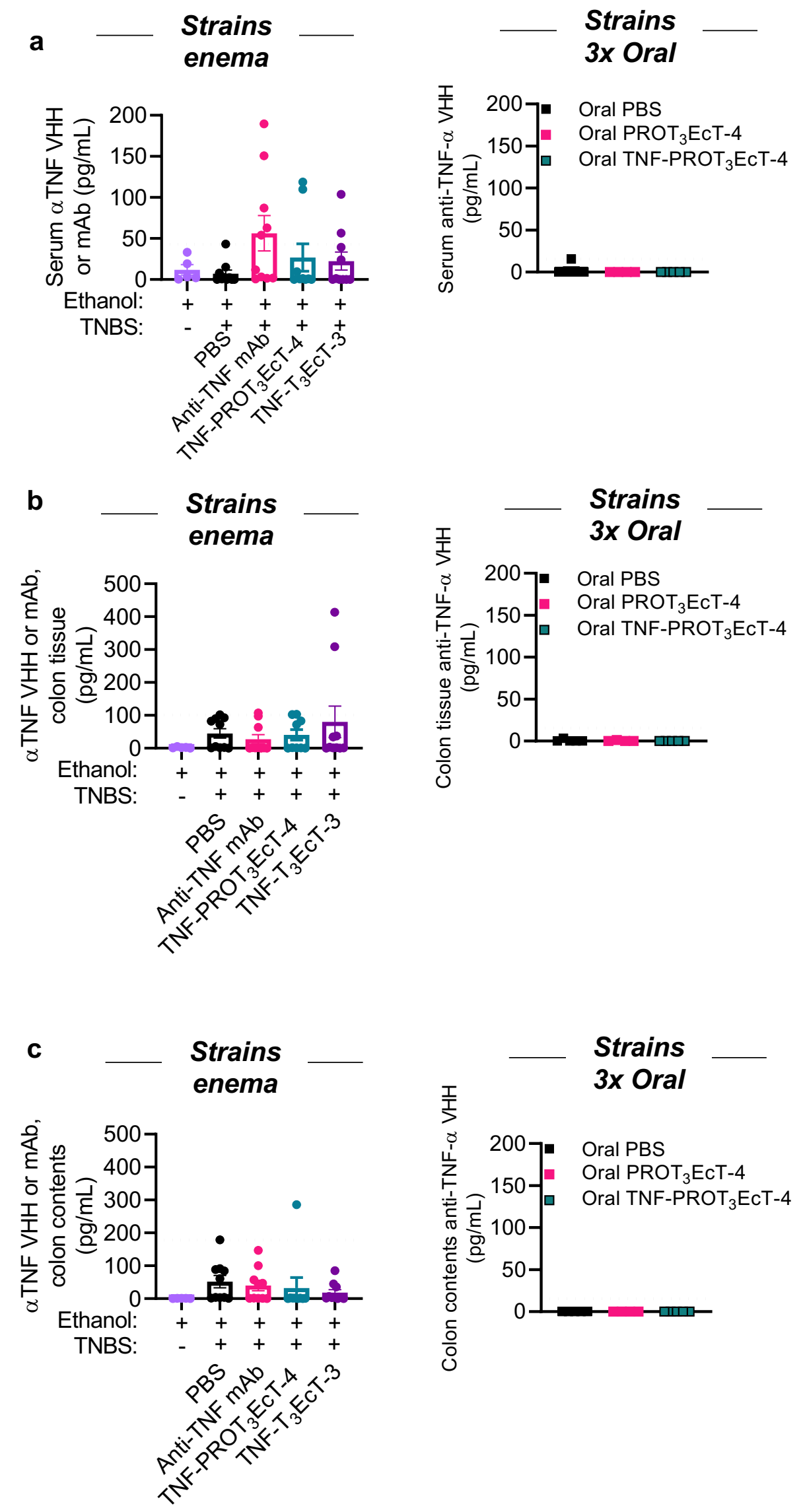